Research Article

\title{
Dynamic Prediction for Accuracy Maintaining Reliability of Superprecision Rolling Bearing in Service
}

\author{
Liang Ye $\mathbb{D},{ }^{1}$ Xintao Xia $\mathbb{D},{ }^{2}$ and Zhen Chang $\mathbb{D}^{3}$ \\ ${ }^{1}$ School of Mechanical Engineering, Northwestern Polytechnical University, Xi'an 710072, China \\ ${ }^{2}$ Mechatronical Engineering College, Henan University of Science and Technology, Luoyang 471003, China \\ ${ }^{3}$ Hangzhou Bearing Test \& Research Center, Hangzhou 310022, China \\ Correspondence should be addressed to Xintao Xia; xiaxt1957@163.com
}

Received 20 April 2018; Revised 20 August 2018; Accepted 30 August 2018; Published 1 October 2018

Academic Editor: Chao Tao

Copyright (c) 2018 Liang Ye et al. This is an open access article distributed under the Creative Commons Attribution License, which permits unrestricted use, distribution, and reproduction in any medium, provided the original work is properly cited.

\begin{abstract}
A dynamic prediction method for accuracy maintaining reliability (AMR) of superprecision rolling bearings (SPRBs) in service is proposed by effectively fusing chaos theory and grey system theory and applying stochastic processes. In this paper, the time series of a vibration signal is used to characterize the state information for SPRB, and four runtime data points can be predicted in the future, which depends on four chaotic forecasting models to preprocess the time series. Using the grey bootstrap method and sampling from the four runtime data, a large amount of generated data (GD) are gained to analyze the changes in information on bearing service accuracy. Then, using a predefined accuracy threshold to match the Poisson count for the GD, the estimated value of variation intensity is obtained. Subsequently, with the help of the Poisson process, the dynamic evolution process is forecast in real time for AMR of the SPRB for each step in the future. Finally, according to a novel concept for maintaining relative reliability in an SPRB, the failure degree of a bearing maintaining an optimum accuracy status (BMOAS) is effectively described. Experimental investigation shows that multiple chaotic forecasting methods are accurate and feasible with all relative errors below $15 \%$; the reliability of each step in the future can truly be described, and the prediction results for AMR over the same subseries show good consistency; dynamic monitoring of the health status of SPRB can be realized by the degree to which a BMOAS fails.
\end{abstract}

\section{Introduction}

A superprecision rolling bearing (SPRB) refers to a kind of accurate rolling bearing with abilities over a wide range of speed, high rotary precision, low fever, high rigidity, low vibration, and low noise, which is a key part of the equipment-manufacturing industries. The reliability of SPRB is brought into sharp focus with rapid developments in equipment-manufacturing industries. Maintaining superior accuracy for SPRB in service is the basis of accuracy maintaining in manufacturing equipment. Owing to the influence of various factors, the running accuracy of the bearings is nonstationary degradation, and the reduction process possesses nonlinear dynamic characteristics. Information, such as precision recession trajectory, probability distribution, and accuracy reliability function, varies with the movement process. The prediction problem for accuracy reliability involves the interaction between internal factors and the external environment [1-5]. According to the existing research, the reliability theory of rolling bearings is primarily concerned with fatigue failure and static reliability problems and assumes that the lifetime data obey the Weibull distribution or lognormal distribution [6-8]. However, in practical applications, the lifetime evaluation index of an SPRB rests on accuracy rather than fatigue, which means that the bearing may be far from reaching its fatigue lifetime, while the accuracy has failed. The existing lifetime evaluation systems are not suitable for SPRB, and there is no set of proprietary theories for dynamically predicting the accuracy maintaining reliability (AMR) of SPRB at each point in the future.

Domestic and foreign research studies have made some achievements in the field of reliability prediction for product components, which provide theoretical references and some suggestions for this investigation, although there is no ideal model for AMR. Kim et al. $[9,10]$ considered the complex functional structures and changeable environments 
of research objects, and their performance degradation was often shown to be nonlinear, nonstationary, and nonGaussian, so the reliability of multiple models was fused into a prediction by making full use of the multisource information. Liu et al. [11] researched a data fusion method for health indicators in degradation modeling, and a narrower confidence interval for the reliability was obtained. Bian and Gebraeel $[12,13]$ viewed the first passage time of a degradation signal for a related device as the remaining life of the equipment and then updated the lifetime signal parameters according to the Bayesian theory. Hong et al. [14] proposed an adaptive method for predicting the health status of bearings and used empirical mode decomposition and self-organizing mapping methods to analyze the bearing vibration information and evaluate the health status at different stages. Tan et al. [15] proposed a fault evolution-test dependency model to improve the testable performance of mechanical systems for tracking fault growth, and this model can provide guidance to engineers searching for failure information in time. Xia [16] proposed a forecasting method for product reliability along with performance data, without any prior information on probability distributions and trends. In the existing literature, there is only a little information available on the reliability of SPRB, so predicting accuracy reliability is the bottleneck that restricts the development of superprecision components and advanced technologies, which is a pressing problem that needs to be solved. In order to effectively solve the problem of accuracy reliability in product components, this paper presents a novel concept for accuracy maintaining reliability and the relative reliability of SPRB in service.

Accuracy maintaining reliability of SPRB is the probability of an SPRB maintaining optimum service accuracy during a test or in the practical application and the probability of it not exceeding its accuracy threshold as well. AMR can be expressed as a function, where the values of this function are called the degree of accuracy maintaining reliability. The accuracy maintaining relative reliability (AMRR) is an error in AMR in the future for each step relative to the AMR at its optimal time, which is used to characterize the failure degree of an SPRB in maintaining the optimum service accuracy status. Most attention focused on SPRB concerns its accuracy index, so it is generally necessary to carry out vibration tests on bearings or precision equipment in order to evaluate the service accuracy of an SPRB effectively and then to preprocess and extract the features from the test data for subsequent research. If the vibration data are small, it indicates that the SPRB is running smoothly and maintaining good rotary accuracy; if they are large, it shows that the SPRB is running roughly with a greater axial moving or radial moving on the spindle, which will cause the service accuracy to be greatly reduced. Therefore, by analyzing the vibration information for the SPRB, the accuracy parameters in the future can be elucidated and predicted, and a dynamic prediction model driven by the vibration data can be established for AMR.

Chaos theory is one of the important methods in nonlinear analysis, due to the vibration performance of time series with nonlinear dynamic characteristics. Analyzing the performance characteristics based on the chaos theory makes the present bearing research at a new height and opens up a new field. Chaotic forecasting models $[17,18]$ can realize dynamic prediction of a time series [19] accurately, extract the evolution information of the system in the future, and explore the inherent deterministic rules of the system. The grey bootstrap method $[20,21]$ can transform a small sample of data from poor information into large sample data for traditional statistical theory. The maximum entropy method $[22,23]$ can be used to obtain the probability density of the sample data, whose main idea is that the solution is the most "unbiased" among all feasible solutions. If a large amount of sample data are given, there will be more accurate sample moments and a more authentic probability density. The Poisson process $[24,25]$ can record the probability of a failure event effectively, but it is difficult to obtain the variation intensity. If one of the above theories is applied only, it will be one-sided and cause many limitations, in which a dynamic forecast of reliability cannot be achieved as well. The innovative nature of this paper is the fusion of the above theories skillfully, where they complement each other. The predicted values of four chaotic prediction models provide four data, which consist of small sample data with poor information. The grey bootstrap method can be used for the conversion to a large sample of data for statistical theory, and then the true probability density function can be calculated using the maximum entropy theory; the variation intensity across the large sample of data is found according to a predetermined accuracy threshold; finally, a reliability prediction is realized via the Poisson process, which records the probability of a system before failure.

Based on the process above, the vibration time series of an SPRB is used to characterize the service accuracy information, and multiple dynamic forecasting models from the time series are built to research different aspects of future trends for the SPRB via chaos theory. A large amount of data generated for different aspects are simulated by the grey bootstrap method, and the probability density function of the generated data is constructed using the maximum entropy method, and then the estimated true values and intervals are forecasted according to the given significance level. Meanwhile, the counting principle is used to process the large amount of generated data, and the variation intensity can be acquired. Then, the concept of AMR for SPRB is proposed using the Poisson process to establish the AMR models, and the dynamic evolution process of AMR is predicted in real time. Finally, the novel concept of AMRR is used to characterize the failure degree of an SPRB maintaining its optimum service accuracy status in the future. The results of the investigation could generate new ideas in new fields of product performance or accuracy reliability, break through the limitations of traditional reliability theory, and enrich the development of basic reliability theories. The proposed models can not only realize dynamic prediction of AMR for SPRB at each point in the future but also effectively forecast the true values and intervals for the future status information. Moreover, the models can monitor the failure degree for future runtime accuracy relative to the optimum service accuracy as well. 


\section{Mathematical Models}

2.1. Chaotic Forecasting Models. Suppose the time series $\mathbf{X}$ for the accuracy information of rolling bearings is given by

$$
\mathbf{X}=\left(x_{1}, x_{2}, \ldots, x_{n}, \ldots, x_{N}\right),
$$

where $n$ stands for the $n$th data for the original series $\mathbf{X}$ and $N$ is the number of original data.

According to the theory of phase space reconstruction, the phase trajectory of the time series $\mathbf{X}$ can be obtained as follows:

$$
\begin{aligned}
\mathbf{X}(t) & =(x(t), x(t+\tau), \ldots, x(t+(k+1) \tau), \ldots, x(t+(m-1) \tau)), \\
t & =1,2, \ldots, M, k=1,2, \ldots, m, \\
M & =N-(m-1) \tau,
\end{aligned}
$$

where $t$ is the th phase trajectory; $x(t+(m-1) \tau)$ is the delay value; $m$ is the embedding dimension, which can be obtained by the Cao method; $\tau$ is the delay time that can be calculated by the mutual information method; and $M$ is the number of phase points. Phase space reconstruction is the basis for predicting the future evolution of the accuracy information for superprecision rolling bearings.
Suppose that $\mathbf{X}(M)$ is the center trajectory (viz., the trajectory of prediction started or the phase space trajectory at the end), there are $L$ reference trajectories similar to the center trajectory, and $\mathbf{X}\left(M_{l}\right)$ is the lth reference trajectory. The chaotic dynamic prediction can be obtained as follows.

2.1.1. Adding-Weight Zero-Rank Local-Region Forecasting Method. According to the theory of phase space reconstruction, the phase trajectory of the time series $\mathbf{X}$ for the accuracy information can be obtained as follows:

$$
\begin{array}{r}
\mathbf{X}(t)=(x(t), x(t+\tau), \ldots, x(t+(m-1) \tau)), \\
t=1,2, \ldots, M,
\end{array}
$$

where $M$ stands for the number of points in the reconstructed phase space with $M=N-(m-1) \tau$, in which $N$ stands for the number of original data.

Based on the adding-weight zero-rank local-region method, the evolution rule of the phase trajectory is

$$
\mathbf{X}(M+1)=\frac{\sum_{l=1}^{L} \mathbf{X}\left(M_{l}\right) e^{-k\left(d l-d_{\min }\right)}}{\sum_{l=1}^{L} e^{-k\left(d l-d_{\min }\right)}}, L=m+1,
$$

with

$$
d l=\sqrt{\left(x(M)-x\left(M_{l}\right)\right)^{2}+\left(x(M+\tau)-x\left(M_{l}+\tau\right)\right)^{2}+\cdots+\left(x(M+(m-1) \tau)-x\left(M_{l}+(m-1) \tau\right)\right)^{2}},
$$

where $\mathbf{X}(M+1)$ is the prediction result; $d l$ is the Euclidean distance between $\mathbf{X}(M)$ and $\mathbf{X}\left(M_{l}\right) ; d_{\text {min }}$ is the minimum value of $d l ; k$ is the prediction parameter, usually $k=1$; and $L$ is the number of reference trajectories.

The detailed steps are the following:

(1) Using the zero-mean method to preprocess the time series $\mathbf{X}$, the sequence $x(t)$ is obtained with $t=1,2, \ldots, N$

(2) Reconstructing the phase space

(3) Finding $L$ reference trajectories expressed as $\mathbf{X}(M)_{\text {reference }}=\left\{\mathbf{X}\left(M_{1}\right), \mathbf{X}\left(M_{2}\right), \ldots, \mathbf{X}\left(M_{L}\right)\right\}$ which are the most proximal points of the center trajectory $\mathbf{X}(M)$, and $\mathbf{X}(M)_{\text {reference }}$ can be obtained by using the Euclidean distance (Equation (5))

(4) Acquiring the prediction results $X(M+1)$

2.1.2. One-Rank Local-Region Forecasting Method. The most proximal points $\mathbf{X}(M)_{\text {reference }}$ around the center trajectory $\mathbf{X}(M)$ are fitted by the linear model of $\mathbf{X}(M+1)=$ $a+b \mathbf{X}(M)$, with

$$
\left[\begin{array}{c}
\mathbf{X}\left(M_{1}+1\right) \\
\mathbf{X}\left(M_{2}+1\right) \\
\cdots \\
\mathbf{X}\left(M_{L}+1\right)
\end{array}\right]=a+b\left[\begin{array}{c}
\mathbf{X}\left(M_{1}\right) \\
\mathbf{X}\left(M_{2}\right) \\
\cdots \\
\mathbf{X}\left(M_{L}\right)
\end{array}\right]
$$

where the points of $\mathbf{X}\left(M_{1}\right), \mathbf{X}\left(M_{2}\right), \ldots, \mathbf{X}\left(M_{L}\right)$ are the proximal points of the center trajectory $\mathbf{X}(M)$.

The coefficients $a$ and $b$ can be solved by the least-squares method, so $\mathbf{X}(M+1)$ is obtained according to the formula $\mathbf{X}(M+1)=a+b \mathbf{X}(M)$, and then the prediction value is efficiently separated.

2.1.3. Adding-Weight One-Rank Local-Region Forecasting Method. Compared with the one-rank local-region forecasting method, the adding-weight one-rank local-region forecasting method considers the influence weight between each proximal point and the center point; namely, the weight term is added, and the weight is as follows:

$$
P_{l}=\frac{e^{-k\left(d l-d_{\min }\right)}}{\sum_{l=1}^{L} e^{-k\left(d l-d_{\min }\right)}},
$$

where $k$ is a forecasting parameter, generally $k=1$.

The linear fitting of the one-rank local-region can be given by

$$
\mathbf{X}\left(M_{l}+1\right)=a \mathbf{R}+b \mathbf{X}\left(M_{l}\right)
$$

with $\mathbf{R}=[1,1, \ldots, 1]^{T}$.

Using the least-adding-squares method to solve the coefficients $a$ and $b$,

$$
\sum_{i=1}^{M} P_{l}\left[\mathbf{X}\left(M_{l}+1\right)-a-b \mathbf{X}\left(M_{l}\right)\right]^{2}=\min ,
$$


with

$$
\left\{\begin{array}{l}
\sum_{l=1}^{L} P_{l}\left[\mathbf{X}\left(M_{l}+1\right)-a-b \mathbf{X}\left(M_{l}\right)\right]=0 \\
\sum_{l=1}^{L} P_{l}\left[\mathbf{X}\left(M_{l}+1\right)-a-b \mathbf{X}\left(M_{l}\right)\right] \mathbf{X}\left(M_{l}\right)=0
\end{array}\right.
$$

viz.,

$$
\left\{\begin{array}{l}
a \sum_{l=1}^{M} P_{l} \mathbf{X}\left(M_{l}\right)+b \sum_{l=1}^{L} P_{i} \mathbf{X}^{2}\left(M_{l}\right)=\sum_{l=1}^{L} P_{i} \mathbf{X}\left(M_{l}\right) \mathbf{X}\left(M_{l}+1\right) \\
a+b \sum_{l=1}^{L} P_{l} \mathbf{X}\left(M_{l}\right)=\sum_{l=1}^{L} P_{l} \mathbf{X}\left(M_{l}+1\right)
\end{array}\right.
$$

The coefficients $a$ and $b$ are acquired by solving equations, and the prediction value is obtained by Equation (8).

2.1.4. Improved Adding-Weight One-Rank Local-Region Forecasting Method. The forecasting method for the improved adding-weight one-rank local-region is proposed based on the adding-weight one-rank local-region method. The difference between the two is the definition of correlation between the center trajectory $\mathbf{X}(M)$ and the proximal points or reference trajectories $\mathbf{X}\left(M_{l}\right)$ : the correlation of the proximal points of the adding-weight one-rank local-region prediction method is defined by the Euclidean distance, and the correlation of the improved method is defined by the cosine value of the angle, with

$$
\cos (l)=\frac{\sum_{l=1}^{L}\left(\mathbf{X}(M), \mathbf{X}\left(M_{l}\right)\right)}{\sqrt{\left(\sum_{l=1}^{L} \mathbf{X}^{2}(M)\right)}\left(\sum_{l=1}^{L} \mathbf{X}^{2}\left(M_{l}\right)\right)},
$$

where $\cos (l)$ is the cosine value of the angle between phase points $\mathbf{X}(M)$ and $\mathbf{X}\left(M_{l}\right)$; thus, the calculation process of the improved adding-weight one-rank local-region method is similar to that of the adding-weight one-rank local-region method; namely, only the Euclidean distance $d l$ is changed into the $\cos (l)$.

2.2. Grey Bootstrap Method. The grey bootstrap method can transform a small sample of data from poor information into large sample data for traditional statistical theory by combining the $\operatorname{GM}(1,1)$ method and bootstrap method in the grey system theory. The $\operatorname{GM}(1,1)$ method weakens the randomness of sample data obviously and generates into random numbers regularly, which makes the accumulative generation and data fusion by optimizing the parameters of differential equations. Moreover, it does not alter the probability density function of sample data, so the grey bootstrap method by using $\operatorname{GM}(1,1)$ can estimate the predicted values of small sample data accurately and effectively.

Using the above four prediction models: adding-weight zero-rank local-region, one-rank local-region, addingweight one-rank local-region, and improved adding-weight one-rank local-region, four service accuracy information points for the $\xi$ th step predicted backward can be forecasted for a superprecision rolling bearing, with the vector $\mathbf{Y}(\xi)$ expressed as follows:

$$
\begin{array}{r}
\mathbf{Y}(\xi)=\left(y_{\xi}(1), y_{\xi}(2), y_{\xi}(3), y_{\xi}(4)\right)=\left(y_{\xi}(u)\right), \\
u=1,2, \ldots, 4, \xi=1,2, \ldots, \psi,
\end{array}
$$

where $y_{\xi}(u)$ is the $u$ th data in $\mathbf{Y}$ for the $\xi$ th step and $\psi$ is the maximum value of the step number predicted backward for four prediction models.

In order to meet $y_{\xi}(u) \geq 0$ according to the grey model $\operatorname{GM}(1,1)[26]$, if $y_{\xi}(u)<0$ in Equation (13), then a constant $c$ should be selected, making $y_{\xi}(u)+c \geq 0$. Therefore, in the actual analysis, $\mathbf{Y}$ is expressed as follows:

$$
\mathbf{Y}(\xi)=\left(y_{\xi}(u)+c\right), \quad u=1,2, \ldots, 4, \xi=1,2, \ldots, \psi
$$

Using the bootstrap method [26], $B$ simulation samples of size $q$, namely, the bootstrap resampling samples $\mathbf{V}_{\text {bootstrap' }}$ can be obtained by an equiprobable sampling with replacement from Equation (14), as follows:

$$
\mathbf{V}_{\text {bootstrap }}=\left(\mathbf{V}_{1}, \mathbf{V}_{2}, \ldots, \mathbf{V}_{b}, \ldots, \mathbf{V}_{B}\right) \text {, }
$$

where $\mathbf{V}_{b}$ is the $b$ th bootstrap resampling sample and $B$ is the times of bootstrap resampling and also the number of bootstrap samples, with

$$
\mathbf{V}_{b}=\left[v_{b}(g)\right],
$$

where $g=1,2, \ldots, q$ and $b=1,2, \ldots, B$.

According to the grey model GM $(1,1)$, suppose the firstorder accumulated generating operator (1-AGO) of $\mathbf{V}_{b}$ is given by

$$
\mathbf{Y}_{b}=\left[y_{\xi b}(u)\right]=\sum_{j=1}^{g} v_{\xi b}(j) .
$$

The grey generated model based on Equation (17) can be described as the differential equation as follows:

$$
\frac{d y_{\xi b}(u)}{d u}+c_{1} y_{\xi b}(u)=c_{2}
$$

where $u$ is the time variable and $c_{1}$ and $c_{2}$ are the coefficients to be estimated.

Use the increment to replace the differential, viz.,

$$
\frac{d y_{\xi b}(u)}{d u}=\frac{\Delta y_{\xi b}(u)}{\Delta u}=y_{\xi b}(u+1)-y_{\xi b}(u)=v_{\xi b}(u+1),
$$

where $\Delta u$ is equal to the unit interval, 1. Furthermore, assume the generated vector of the mean series as follows:

$$
\mathbf{Z}_{b}=\left[z_{b}(u)\right]=\left\lfloor 0.5 y_{\xi b}(u)+0.5 y_{\xi b}(u-1)\right\rfloor .
$$

The least-squares solution for Equation (18) with the initial condition $y_{\xi} b(1)=v_{\xi} b(1)$ is given by

$$
\widehat{y}_{\xi b}(q+1)=\left(v_{\xi b}(1)-\frac{c_{2}}{c_{1}}\right) e^{-c_{1} q}+\frac{c_{2}}{c_{1}},
$$


where the coefficients $c_{1}$ and $c_{2}$ are as follows:

$$
\left(c_{1}, c_{2}\right)^{T}=\left(\mathbf{D}^{T} \mathbf{D}\right)^{-1} \mathbf{D}^{T}\left(\mathbf{V}_{b}\right)^{T},
$$

with

$$
\begin{aligned}
\mathbf{D} & =\left(-\mathbf{Z}_{b}, \mathbf{I}\right)^{T}, \\
\mathbf{I} & =(1,1, \ldots, 1) .
\end{aligned}
$$

According to the inverse AGO, the $b$ th generated data are expressed as follows:

$$
\widehat{v}(q+1)=\hat{y}_{\xi b}(q+1)-\hat{y}_{\xi b}(q)-c .
$$

Therefore, $B$ generated data for the service accuracy can be obtained as the following vector:

$$
\begin{aligned}
\mathbf{Y}_{B} & =\left(w_{1}, w_{2}, \ldots, w_{b}, \ldots, w_{B}\right) \\
& =\left(\widehat{v}_{1}(q+1), \widehat{v}_{2}(q+1), \ldots, \widehat{v}_{b}(q+1), \ldots, \widehat{v}_{B}(q+1)\right),
\end{aligned}
$$

where $w_{b}$ is the $b$ th generated data.

\subsection{Maximum Entropy Principle}

\subsubsection{Solving the Probability Density Function.} Transmuting the generated data $\mathbf{Y}_{B}$ for the service accuracy in Equation (25) into continuous information, the expression of maximum entropy is defined as follows:

$$
H(w)=-\int_{-\infty}^{+\infty} p(w) \ln p(w) d w,
$$

where $p(w)$ is the probability density function of the data series $\mathbf{Y}_{B}$. By the maximum entropy principle, the optimal estimation of the density function based on sample information can be obtained, and the main idea of maximum entropy is that the solution is the most "unbiased" among all feasible solutions, given as follows:

$$
H(w)=-\int_{S} p(w) \ln p(w) d w \longrightarrow \max ,
$$

where $S$ represents the integral interval, namely, the feasible region for the random variable $w$.

It satisfies the constraint condition:

$$
\int_{S} w^{j} p(w) d w=m_{j}, \quad j=0,1,2, \ldots, \beta, m_{0}=1,
$$

where $m_{j}$ stands for the $j$ th-order origin moment, with $\beta$ for the highest origin moment order.

The entropy can reach its maximum by adjusting $p(w)$, and the probability density function $p(w)$ can be obtained by using the Lagrange multiplier method, as follows:

$$
p(w)=\exp \left(\lambda_{0}+\sum_{j=1}^{\beta} \lambda_{j} w^{j}\right),
$$

where $\lambda_{0}, \lambda_{1}, \ldots, \lambda_{\alpha}$ are Lagrange multipliers and $w$ is a random variable for service accuracy, with

$$
\begin{aligned}
& m_{j}=\frac{\int_{S} w^{j} \exp \left(\sum_{j=1}^{\beta} \lambda_{j} w^{j}\right) d w}{\int_{S} \exp \left(\sum_{j=1}^{\beta} \lambda_{j} w^{j}\right) d w}, \\
& \lambda_{0}=-\ln \left(\int_{S} \exp \left(\sum_{j=1}^{\beta} \lambda_{j} w^{j}\right) d w\right) .
\end{aligned}
$$

Equation (29) is the probability density function of generated data $\mathbf{Y}_{B}$, which is constructed by using the maximum entropy principle. With the help of function $p(w)$, the true value and the confidence interval of the generated series can be implemented as follows.

2.3.2. Parameter Estimation. According to the probability density function $p(w)$ of the random variable $w$, the estimated true value $X_{0}$ of series $\mathbf{Y}_{B}$ is given by

$$
X_{0}=\int_{-\infty}^{+\infty} w p(w) d w .
$$

If the real number $\alpha \in(0,1)$ exists, then the probability of $w_{\alpha}$ is given by

$$
P\left(X<X_{\alpha}\right)=\int_{-\infty}^{w_{\alpha}} p(w) d w=\alpha,
$$

where $w_{\alpha}$ is the $\alpha$ quantile of the density function $p(w)$ and $\alpha$ is the significance level.

For the bilateral quantile, the probability is as follows:

$$
\begin{aligned}
& P\left(X<X_{\mathrm{U}}\right)=\frac{\alpha}{2}, \\
& P\left(X \geq X_{\mathrm{L}}\right)=\frac{\alpha}{2},
\end{aligned}
$$

where $X_{\mathrm{U}}$ and $X_{\mathrm{L}}$ are the upper and lower boundaries of the generated series $\mathbf{Y}_{B}$, respectively, and $\left[X_{\mathrm{L}}, X_{\mathrm{U}}\right]$ is the confidence interval under the $\alpha$ level.

Therefore, four points for accuracy information for superprecision rolling bearings are fused by effectively combining the grey bootstrap method with the maximum entropy method, and then the true value $X_{0}$ and the interval $\left[X_{\mathrm{L}}, X_{\mathrm{U}}\right]$ for its accuracy information can be predicted for each moment in the future.

\subsection{Poisson Process}

2.4.1. Counting Process. If the accuracy generated sequence $\mathbf{Y}_{B}$ (in Equation (25)) for superprecision rolling bearings has $\mu$ data over the accuracy threshold $h$, namely, there are $\mu$ data falling out of the interval $[0, h]$ of optimum service accuracy, the estimated value for variation intensity $\theta$ of the generated sequence $\mathbf{Y}_{B}$ is expressed as follows:

$$
\theta=\frac{\mu}{B}
$$

Variation intensity refers to the frequency of accuracy amplitude for superprecision rolling bearings exceeding the optimum accuracy interval, which is the important and characteristic parameter for the runtime accuracy affecting 
the variation process, and it varies with the accuracy threshold.

2.4.2. Dynamic Prediction for Accuracy Maintaining Reliability. Any counting process can be described using the Poisson process as follows:

$$
Q=\exp (-\theta i) \frac{(\theta i)^{e}}{e !}
$$

where $i$ stands for the time variable with $i=1,2,3, \ldots(i \geq 1)$; $\theta$ for the variation intensity; $e$ for the number of occurring failure events with $e=0,1,2,3, \ldots$, namely, the serious variation in service accuracy that may cause the bearing failure; and $Q$ for the probability of failure events occurring $e$ times. Thus, the reliability $R$ for failure events can be obtained using the Poisson process.

When solving for the accuracy maintaining reliability $R$ for superprecision rolling bearings in service, let $e=0$; viz., the frequency of occurrence of accuracy failure is zero, which indicates that $R$ is the probability before the accuracy failure occurs. Let $i=1$, which indicates that $R$ is the accuracy maintaining reliability in real time; namely, it is the probability of the service accuracy being maintained in the interval $[0, h]$ at optimum accuracy for the currently generated sequence $\mathbf{Y}_{B}$. According to Equation (35), reliability can be expressed as follows:

$$
R(\theta)=\exp (-\theta)
$$

where $R(\theta)$ stands for the probability of the running status maintaining optimum accuracy for the superprecision rolling bearings in service.

Then, the reliability $R(\theta)$ of sequence $\mathbf{Y}_{B}$ is only a function of the variation intensity $\theta$, and $\theta$ can be obtained by Equation (34). In a concrete implementation, if the accuracy maintaining reliability (AMR) $R(\theta)$ is not less than $90 \%$, then the reliability of bearing service accuracy is deemed to be good and its possibility of maintaining an optimum service accuracy state is great. If the reliability $R(\theta)$ is less than $90 \%$ and not less than $80 \%$, the reliability of bearing service accuracy is deemed to be general and its possibility of maintaining an optimum service accuracy state is gradually being reduced. If the reliability $R(\theta)$ is less than $80 \%$ and not less than $50 \%$, the reliability of bearing service accuracy is deemed to be low and the possibility of maintaining an optimum service accuracy state is poor. If the reliability $R(\theta)$ is less than $50 \%$, the bearing service accuracy can be regarded as failed.

\subsubsection{Service Accuracy Maintaining the Relative Reliability} $(S A M R R)$. The variation intensity $\theta_{1}$ for the optimum service accuracy is obtained at the optimal time (generally the initial runtime) for a superprecision rolling bearing (SPRB), and the variation intensity of service accuracy at other times is expressed as $\theta_{\eta}$ with $\eta=2,3,4, \ldots$.. According to the concept of relative error in measurement theory, service accuracy maintaining the relative reliability $d(\eta)$ of an SPRB is obtained to characterize the failure degree of maintaining runtime optimum accuracy.

$$
d(\eta)=\frac{R\left(\theta_{\eta}\right)-R\left(\theta_{1}\right)}{R\left(\theta_{1}\right)} \times 100 \%,
$$

where $R\left(\theta_{1}\right)$ stands for the SPRB reliability of maintaining optimal service accuracy at the optimal time, $R\left(\theta_{\eta}\right)$ for others' reliability of maintaining optimum service accuracy at different future times, and $d(\eta)$ for the failure degree of SPRB in maintaining an optimum service status at different future times.

\subsubsection{Evaluation of the Failure Degree of the Optimum} Service Accuracy State. Accuracy maintaining reliability (AMR) of an SPRB is the probability of it maintaining the optimum service accuracy status and the values of service accuracy not exceeding the accuracy threshold. The concept of AMR is proposed using the Poisson process to establish the AMR models and predict the dynamic evolution process of AMR in real time. The variation intensity of the generated sequence $\mathbf{Y}_{B}$ is obtained by recording the number of sample data falling out of the interval $[0, h]$ of optimum service accuracy; then, based on the Poisson process, the estimated value for the AMR of an SPRB can be obtained according to the Equations (35) and (36). Therefore, by analyzing the vibration information of the SPRB, the accuracy parameters in the future can be elucidated and predicted, and a dynamic prediction model driven by the vibration data can be established for AMR.

The accuracy maintaining relative reliability (AMRR) is an error in AMR in the future for each step relative to the AMR at its optimal time, which is used to characterize the failure degree of an SPRB in maintaining the optimum service accuracy status. The basic principle of service accuracy classification for an SPRB is as follows:

(1) According to the principle of significance in hypothesis testing, if SAMRR $d(\eta)$ of SPRB is not less than $0 \%$, which shows that the AMR at this assessment time is not less than AMR at the optimum time, it cannot deny that the service accuracy of the SPRB has reached its optimal state; otherwise, it can deny that the service accuracy has achieved its optimal state.

(2) When SAMRR $d(\eta)$ of SPRB is less than $0 \%$, if the absolute value of relative error $d(\eta)$ is in $(0 \%, 10 \%]$, this indicates that the error between the evaluation value and the optimum value is very small. If the absolute value of relative error is in $(10 \%, 20 \%]$, this indicates that the error between the evaluation value and the optimum value is gradually increasing. If the absolute value of relative error is greater than $20 \%$, this indicates that the error between the evaluation value and the optimum value is very large.

Based on that, the service accuracy of an SPRB is divided into S1, S2, S3, and S4 for a total of four levels:

S1: if relative reliability $d(\eta) \geq 0 \%$ of the SPRB, it indicates the bearing service accuracy at a future time reaches the optimum, and the accuracy state has almost no failure possibility. 
S2: if relative reliability $d(\eta) \in[-10 \%, 0 \%)$ of the SPRB, it indicates the bearing service accuracy at a future time is normal, and the failure possibility of the accuracy state is very small.

S3: if relative reliability $d(\eta) \in[-20 \%,-10 \%)$ of the SPRB, it indicates the bearing service accuracy at a future time is gradually becoming worse, and the failure possibility of the accuracy state is gradually increasing.

S4: if relative reliability $d(\eta)<-20 \%$ of the SPRB, it indicates the bearing service accuracy at a future time is worse, and the failure possibility of the accuracy state is very large.

According to the four levels of service accuracy for an SPRB, the evolution process of the failure degree in maintaining an optimum accuracy state can be predicted effectively. SAMRR of an SPRB really refers to the attenuation degree in AMR in the future time relative to the optimum service accuracy state. The negative value of the SAMRR indicates that the bearing state has attenuation; namely, AMR currently is less than AMR at the optimum time, and the positive value indicates no attenuation. The smaller the relative reliability $d(\eta)$ is, the worse the bearing service accuracy is and the larger the failure probability of the optimum service accuracy status is.

Therefore, the period of $d(\eta)=-20 \%$ is the critical time where bearing service accuracy becomes poor. Putting appropriate measures in place before the critical time can avoid serious safety accidents that are caused by a state failure in optimum service accuracy.

2.5. Basic Modeling Idea. A variety of mathematical methods including chaotic prediction methods, the grey bootstrap method, the maximum entropy principle, and the Poisson process are used during theoretical modeling. Each model does not exist or function here as an isolated single model but rather function by complementing and interlocking with each other, which breaks through the limitations whereby one method can only solve a certain type of problem. The idea works as follows:

Step 1. Based on a time series $\mathbf{X}$ of accuracy information, using the Cao method to obtain the embedding dimension $m$ and using the mutual information method to acquire the time delay $\tau$, the phase space is reconstructed.

Step 2. According to the phase space reconstruction to obtain the phase trajectory of time series $\mathbf{X}$, with the help of four chaotic dynamic prediction models including adding-weight zero-rank local-region, one-rank local-region, adding-weight one-rank local-region, and improved adding-weight one-rank local-region, four forecast values for each step in the future are obtained, composing the small sample $\mathbf{Y}$ of size four.

Step 3. With the help of the grey bootstrap method, a large sample $\mathbf{Y}_{B}$ is generated from the small sample $\mathbf{Y}$ to solve the accurate probability density function.
Step 4. Let the large sample $\mathbf{Y}_{B}$ be continuous data, and solve the sample moment of each order, according to the maximum entropy principle to obtain the probability density function, and then calculate the true value $X_{0}$ and the interval $\left[X_{\mathrm{L}}, X_{\mathrm{U}}\right]$ under the $\alpha$ level for each step in the future, and the prediction of accurate information is achieved.

Step 5. According to the given accuracy threshold $h$, find out the number $\mu$ of $\mathbf{Y}_{B}$ outside the optimum service accuracy interval $[0, h]$ and then obtain the variation intensity $\theta$ for the prediction results of each step; then, accuracy maintaining reliability $R(\theta)$ of an SPRB for each step in the future is achieved according to the Poisson formula.

Step 6. By the new concept of service accuracy maintaining the relative reliability, the relative reliability $d(\eta)$ of an SPRB is obtained to characterize the degree of failure of future runtime accuracy in maintaining the optimum status.

2.6. Fault Declaration. The innovative nature of this paper is fusing the chaos theory and grey system theory and applying stochastic processes skillfully, where they complement each other. Certainly, benefits and limits of the fusion approach proposed exist. It can realize the dynamic prediction of a time series with nonlinear dynamic characteristics accurately and effectively, extract the evolution information of the system in the future, and explore the inherent deterministic rules of the system. Furthermore, for small sample data with poor information, the fusion approach weakens the randomness of sample data obviously and generates into random numbers regularly, which does not alter the probability density function of sample data as well. Besides, for all the potential fault types, the fusion approach proposed is applicable to the performance data sample, without any prior information on probability distributions and trends.

The only drawback is that the sensitivity to the initial values of sample data is great for bearing performance owing to the use of chaos theory, and the selection of the accuracy threshold has an effect on calculating variation intensity.

Many fault types do exist and have an effect on the running accuracy of the bearings, which can be reflected in the performance data collected. The evaluation models realize dynamic prediction of AMR and monitor the failure degree of the optimum service accuracy status based on the performance data collected in the experiment, so they are applicable to all the potential fault types.

\section{Experimental Results and Discussion}

This is a strength test of the lifetime accuracy for superprecision rolling bearings, and the test machine model is an ABLT-1A, which mainly includes the test head seat, test head, transmission system, loading system, lubrication system, and computer control system. The test material is a $\mathrm{P} 4$ bearing $\mathrm{H} 7008 \mathrm{C}$ provided by SKF, and this kind of bearing has a moving grade, which can almost meet the 
accuracy requirements of $\mathrm{P} 2$ grade. The research is conducted at a room temperature of $26^{\circ} \mathrm{C}$, a relative humidity of $53 \%$, a motor speed of $4950 \mathrm{r} / \mathrm{min}$, and a radial load of $13.2 \mathrm{kN}$. Test time and bearing vibration information are automatically recorded by the computer control system: the sampling frequency of bearing vibration amplitude is $5 \mathrm{kHz}$, and the root mean square values of vibration amplitudes are obtained by each $10 \mathrm{~min}$; then vibration data are collected by the computer with unit in $\mathrm{m} \cdot \mathrm{s}^{-2}$; namely, the bearing vibration signal is the root mean square value of vibration amplitudes within 10 minutes. From the beginning of the test, if significant variation occurs in the bearing ring or the roller or even surface fatigue spalling, the vibration value of the test machine will obviously increase and service accuracy will be reduced. If the vibration value reaches a certain value, the motor will stop running, and the experiment will be over.

The computer collects a total of 8010 signals; that is, the experiment lasts for $8010 \times 10 \mathrm{~min}$, and the vibration signal of time series $\mathbf{X}$ obtained for the superprecision rolling bearings is shown in Figure 1. It is not difficult to see that, with the increase in runtime, the vibration value becomes larger, which means that the malignant variation in service accuracy becomes more serious, and then accuracy maintaining reliability will gradually decline. Therefore, the potential evolution process of service accuracy can be described as analyzing the vibration signals of a superprecision rolling bearing and then predicting the future running conditions and accuracy maintaining reliability.

3.1. Chaotic Forecasting of Time Series. The time series $\mathbf{X}$ is divided into four subseries, namely, $\mathbf{X}_{1}(1-2000), \mathbf{X}_{2}(2001-$ 4000), $\mathbf{X}_{3}(4001-6000)$, and $\mathbf{X}_{4}(6001-8000)$, and then the chaotic prediction methods are used to establish the corresponding models for the four subseries, where the prediction step length is 10 steps. By using the original data of 2001-2010 $\left(\mathbf{X}_{1,0}\right), 4001-4010\left(\mathbf{X}_{2,0}\right), 6001-6010\left(\mathbf{X}_{3,0}\right)$, and $8001-8010\left(\mathbf{X}_{4,0}\right)$, the prediction models of the four subseries are verified as feasible and accurate, respectively.

Solving phase space parameters: the mutual information method and the Cao method were used to, respectively, calculate the time delay $\tau$ and the embedding dimension $m$ of the time series of $\mathbf{X}_{1}, \mathbf{X}_{2}, \mathbf{X}_{3}$, and $\mathbf{X}_{4}$, and the results are shown in Table 1.

The calculation of phase space parameters for the time series is the basis of phase space reconstruction, which is helpful for the following chaotic forecasting. Figures $2-5$ are the chaotic prediction results and original data for subseries $\mathbf{X}_{1}, \mathbf{X}_{2}, \mathbf{X}_{3}$, and $\mathbf{X}_{4}$ for the 10 steps in the future, respectively, using the adding-weight zero-rank local-region method (marked as method 1), one-rank local-region method (method 2), adding-weight one-rank local-region method (method 3), and improved adding-weight one-rank localregion method (method 4).

From Figure 2, the variation trends for the four forecasting results of subseries $\mathbf{X}_{1}$ are very similar and relatively stable, with a forecasting value floating around $0.70 \mathrm{~m} \cdot \mathrm{s}^{-2}$, and the difference with the original data is a little large in the

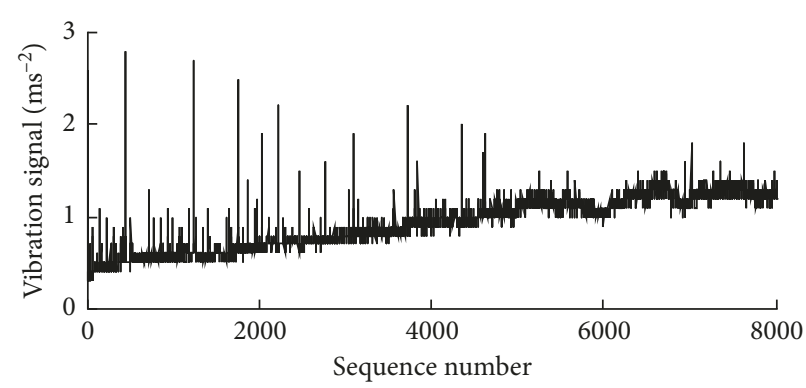

Figure 1: Vibration time series $\mathbf{X}$ of a superprecision rolling bearing.

TABle 1: Phase space parameters of 4 subseries.

\begin{tabular}{lcccc}
\hline Phase space parameters & $\mathbf{X}_{1}$ & $\mathbf{X}_{2}$ & $\mathbf{X}_{3}$ & $\mathbf{X}_{4}$ \\
\hline$\tau$ & 3 & 1 & 1 & 2 \\
$m$ & 16 & 7 & 9 & 9 \\
\hline
\end{tabular}

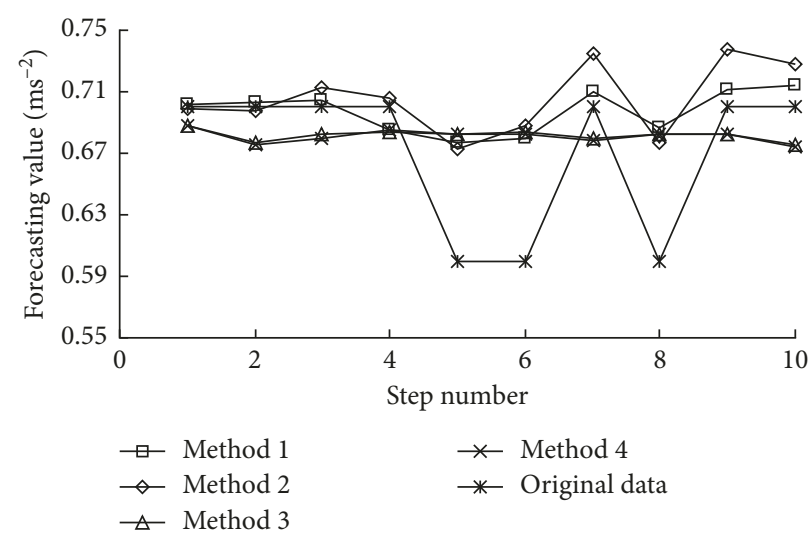

FIgURE 2: Chaotic prediction results for subseries $\mathbf{X}_{1}$.

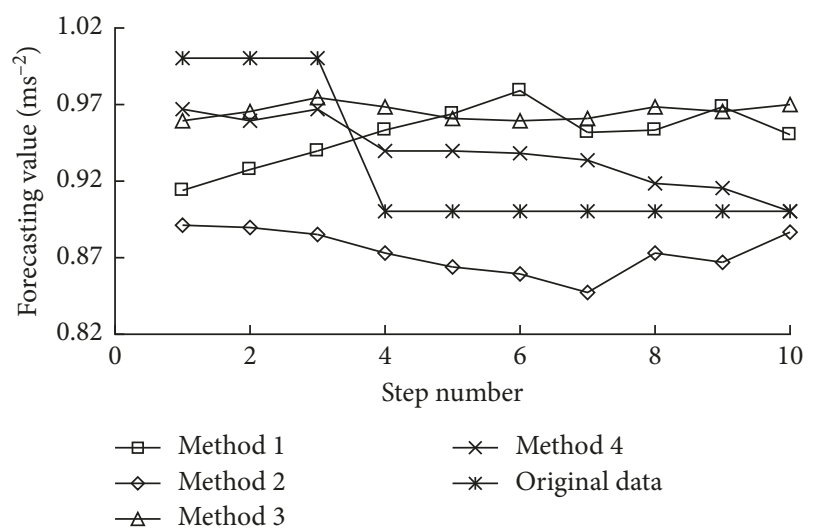

Figure 3: Chaotic prediction results for subseries $\mathbf{X}_{2}$.

$5^{\text {th }}, 6^{\text {th }}$, and $8^{\text {th }}$ steps, but the maximum difference is only about $0.10 \mathrm{~m} \cdot \mathrm{s}^{-2}$. From Figure 3, the difference in subseries $\mathbf{X}_{2}$ between the four forecasting results and the original data is very small, and the difference is slightly large in the first three steps of the one-rank local-region method (method 2), but only about $0.10 \mathrm{~m} \cdot \mathrm{s}^{-2}$. From Figure 4, the original 


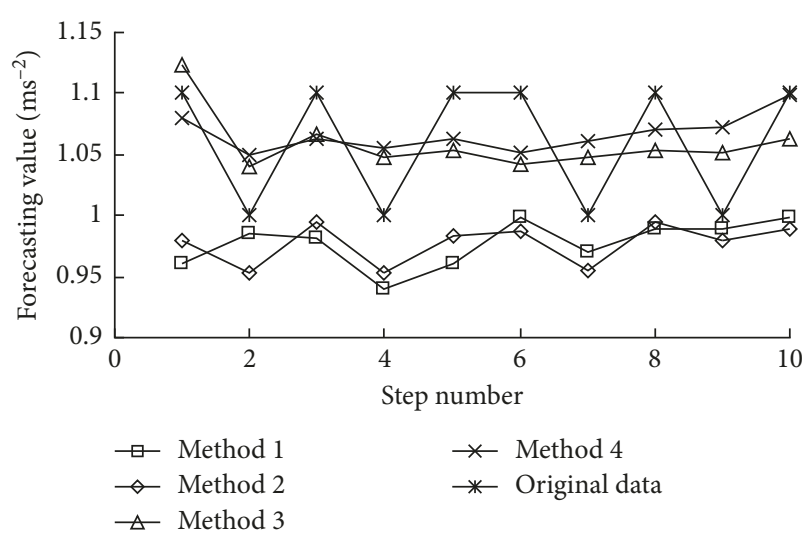

FIgURE 4: Chaotic prediction results for subseries $\mathbf{X}_{3}$.

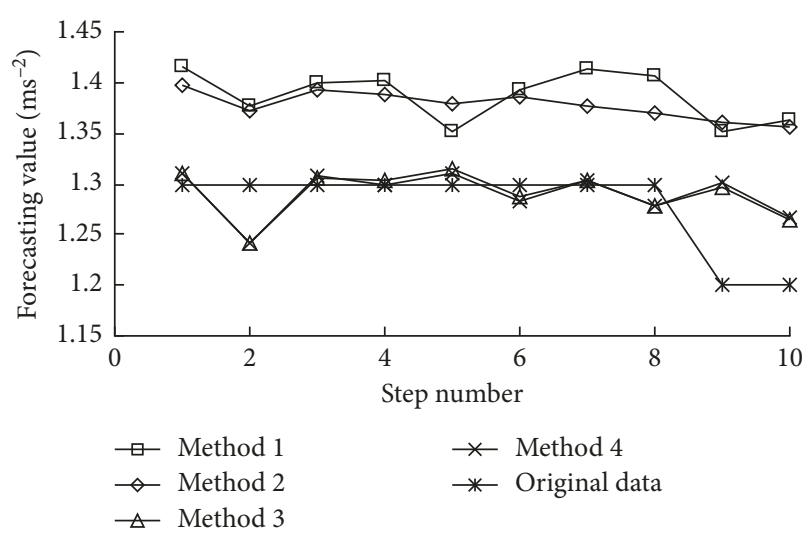

FIgURE 5: Chaotic prediction results for subseries $\mathbf{X}_{4}$.

data from subseries $\mathbf{X}_{3}$ jump between $1 \mathrm{~m} \cdot \mathrm{s}^{-2}$ and $1.1 \mathrm{~m} \cdot \mathrm{s}^{-2}$, the forecasting results of the adding-weight zero-rank local-region method (method 1) and the one-rank localregion method (method 2) float around $0.94-1.0 \mathrm{~m} \cdot \mathrm{s}^{-2}$, and the forecasting results of the adding-weight one-rank localregion method (method 3 ) and the improved adding-weight one-rank local-region method (method 4) float around $1.04-1.12 \mathrm{~m} \cdot \mathrm{s}^{-2}$, which shows that the difference between the four prediction results and the actual values is very small. From Figure 5, the prediction results of method 1 and method 2 for subseries $\mathbf{X}_{4}$ are around $1.40 \mathrm{~m} \cdot \mathrm{s}^{-2}$, and the results of method 3 and method 4 are around $1.30 \mathrm{~m} \cdot \mathrm{s}^{-2}$, which shows that the difference between the four prediction results and the actual values is also small. Thus, when the four chaotic forecasting models are used in time-series forecasting, all the differences between the predicted values and actual values are small, and these models are accurate and reliable.

In order to illustrate the validity of the prediction results, the relative errors are calculated between the predicted values and the original data from subseries $\mathbf{X}_{1}, \mathbf{X}_{2}, \mathbf{X}_{3}$, and $\mathbf{X}_{4}$. The absolute values of the relative errors are shown in Figures 6-9.

From Figure 6, it is not difficult to see that the trends in the relative errors from the four forecasting results from subseries $\mathbf{X}_{1}$ are very similar, which shows that the

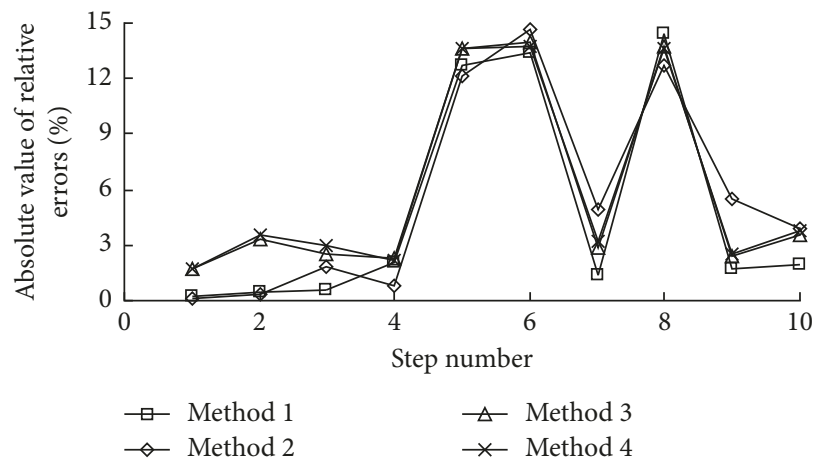

FIgURE 6: Absolute value of the relative errors from the prediction results from subseries $\mathbf{X}_{1}$.

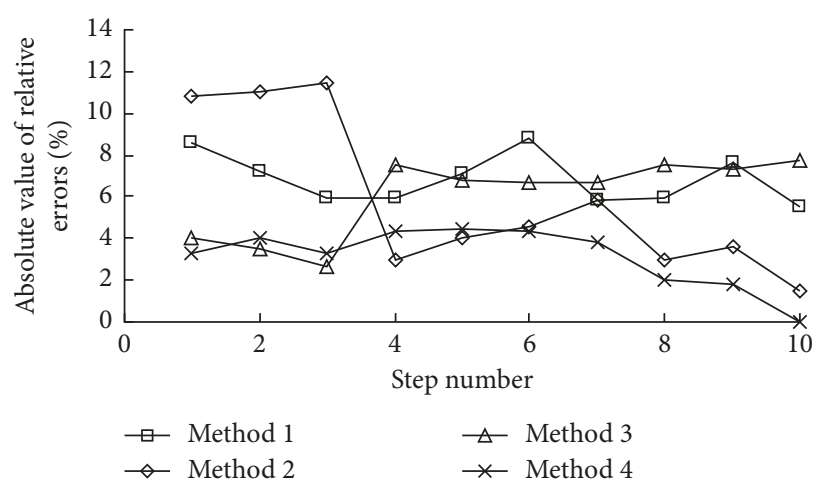

FIgURE 7: Absolute value of the relative errors from the prediction results from subseries $\mathbf{X}_{2}$.

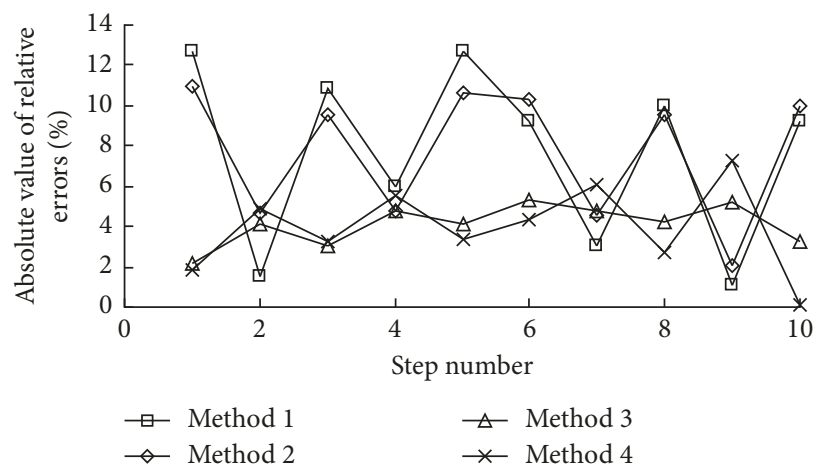

Figure 8: Absolute value of the relative errors from the prediction results from subseries $\mathbf{X}_{3}$.

prediction results have good consistency. The minimum error appearing in the first step of method 2 is $0.13 \%$, showing that the prediction result is very precise, and the maximum error appears in the sixth step of method 2 but is only $14.68 \%$. From Figure 7 , in the prediction models of subseries $\mathbf{X}_{2}$, the predicted results from method 4 are the best, with the prediction error around $0-4.48 \%$. The prediction results from method 2 are somewhat poor, and the maximum prediction error appearing is $11.43 \%$; in the other 2 methods, the prediction errors are not more than $8.8 \%$. From Figure 8, the fluctuation in relative error from the four 


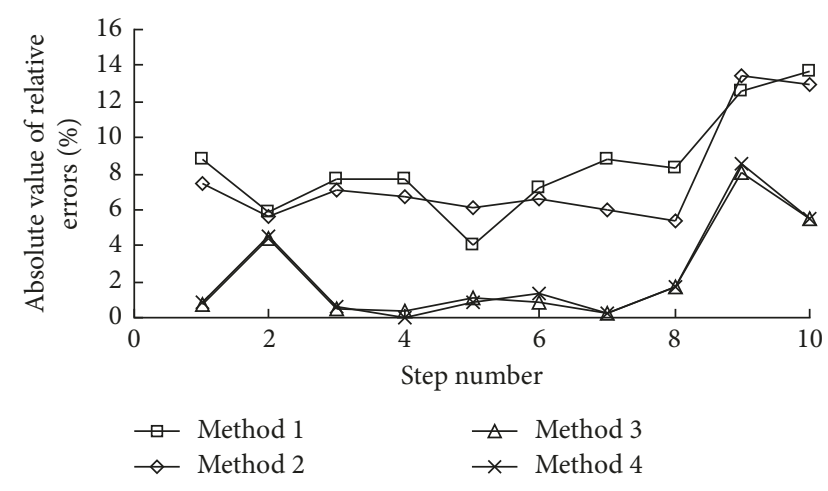

Figure 9: Absolute value of the relative errors from the prediction results from subseries $\mathbf{X}_{4}$.

prediction results from subseries $\mathbf{X}_{3}$ is violent because the original data fluctuation is violent (showing serration), which indicates that the prediction models can reflect the trend change in the original series. The minimum error appearing in method 4 is $0.11 \%$, and the maximum relative error appears in method 1, but is only $12.70 \%$. From Figure 9 , in the prediction models of subseries $\mathbf{X}_{4}$, the prediction results of method 3 and method 4 are better than those of method 1 and method 2, and the maximum relative error appears in method 1 but is only $13.63 \%$.

In summary, the prediction results for the four subseries show that relative errors from the chaotic forecasting models including the adding-weight zero-rank local-region method, one-rank local-region method, adding-weight one-rank local-region method, and improved adding-weight onerank local-region method are all very small and all less than $15 \%$, so the four forecasting models can be applied to practical engineering. However, it is difficult to find out which method is the best or the worst of the four forecasting models for the four subseries because the models' advantages or disadvantages are different in different subseries. A single prediction method can only reflect one facet of service accuracy in the future, and a prediction value for each step is a character of the true value. Only by fusing and eliciting multiple aspects of the information, a forecast of the true value can be realized. The grey bootstrap method and maximum entropy principle are fused to be applied to the four forecasting results in each step: using the grey bootstrap method for sample processing the four forecast results, a large number of generated data are simulated; by the maximum entropy principle, the probability density of the generated data is calculated, the predicted true value is gained, and the prediction interval is then obtained under a given confidence level.

\subsection{True Value Evaluation and Interval Prediction. In the} process of grey bootstrap generation, let the sampling number $q=4$, the times for bootstrap resampling $B=20000$, and the significant level $\alpha=0$. The generated data and probability density of the four prediction values $\mathbf{X}_{1,1}=$ $[0.7013,0.6991,0.6878,0.6883]$ of subseries $\mathbf{X}_{1}$ in the first step are shown in Figures 10 and 11.

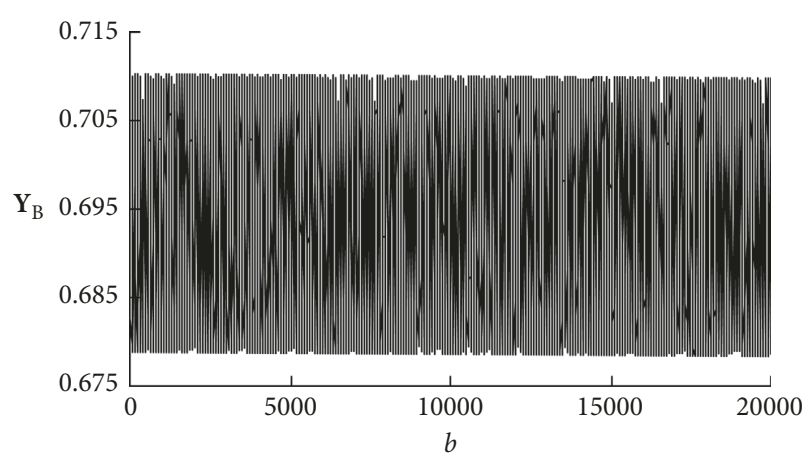

Figure 10: Generated data of $\mathbf{X}_{1,1}$.

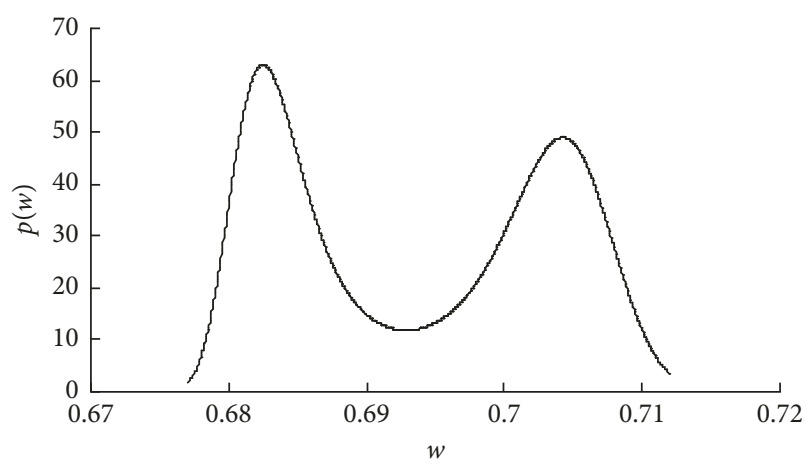

Figure 11: Probability density distribution of $\mathbf{X}_{1,1}$.

Thus, the estimated true value $X_{0}=0.6937$ and the estimated interval $\left[X_{\mathrm{L}}, X_{\mathrm{U}}\right]=[0.6677,0.7121]$ are obtained in the first step of the subseries $\mathbf{X}_{1}$. Then, the estimated true value and the estimated interval can be acquired in the $2^{\text {nd }}$ through $10^{\text {th }}$ steps of subseries $\mathbf{X}_{1}$. Similarly, the estimated true value and the estimated interval can be obtained for the 10 steps in the future for the other subseries of $\mathbf{X}_{2}, \mathbf{X}_{3}$, and $\mathbf{X}_{4}$. The results are shown in Tables 2 and 3 .

From Table 2, each subseries is predicted for 10 steps, namely, the runtime situation of a superprecision rolling bearing is forecast into the future $10 \times 10 \mathrm{~min}$. The vibration performance of subseries $\mathbf{X}_{1}, \mathbf{X}_{2}, \mathbf{X}_{3}$, and $\mathbf{X}_{4}$ is around $0.69 \mathrm{~m} \cdot \mathrm{s}^{-2}, 0.93 \mathrm{~m} \cdot \mathrm{s}^{-2}, 1.01 \mathrm{~m} \cdot \mathrm{s}^{-2}$, and $1.33 \mathrm{~m} \cdot \mathrm{s}^{-2}$ into the future $10 \times 10 \mathrm{~min}$, respectively, showing that the forecast results for each series have good consistency. From Table 3, the difference between the upper and lower limits of each estimated interval is very small, showing that the prediction interval is accurate and reliable. At the same time, referring to the original data in Figures 2-5 of each subseries, the estimated interval $\left[X_{\mathrm{L}}, X_{\mathrm{U}}\right]$ envelopes all the original data; namely, the proposed prediction models can describe the fluctuation in service accuracy of a superprecision rolling bearing effectively and can also realize online monitoring of the dynamic information for the bearing components, so the reliability of the interval prediction reaches $(1-0 / 15) \times$ $100=100 \%$. In order to verify that the true value of the fusion can thoroughly reflect the future situation and accurately forecast the trend, the relative errors between the true value and the actual value are calculated, and the results are shown in Figure 12. 
TABLE 2: Estimated true value $X_{0}$ for each subseries for 10 steps in the future.

\begin{tabular}{lcccc}
\hline Step number & $\mathbf{X}_{1}$ & $\mathbf{X}_{2}$ & $\mathbf{X}_{3}$ & $\mathbf{X}_{4}$ \\
\hline $1^{\text {st }}$ step & 0.6937 & 0.9318 & 1.0387 & 1.3585 \\
$2^{\text {nd }}$ step & 0.6871 & 0.9336 & 1.0046 & 1.3136 \\
$3^{\text {rd }}$ step & 0.6953 & 0.9387 & 1.0289 & 1.3550 \\
$4^{\text {th }}$ step & 0.6907 & 0.9307 & 0.9988 & 1.3471 \\
$5^{\text {th }}$ step & 0.6780 & 0.9277 & 1.0136 & 1.3418 \\
$6^{\text {th }}$ step & 0.6841 & 0.9283 & 1.0192 & 1.3371 \\
$7^{\text {th }}$ step & 0.7018 & 0.9184 & 1.0084 & 1.3487 \\
$8^{\text {th }}$ step & 0.6815 & 0.9265 & 1.0252 & 1.3331 \\
$9^{\text {th }}$ step & 0.7068 & 0.9266 & 1.0222 & 1.3269 \\
$10^{\text {th }}$ step & 0.6981 & 0.9264 & 1.0343 & 1.3166 \\
\hline
\end{tabular}

From Figure 12, the true value after fusing the four prediction values in each step can be seen and its prediction accuracy is fairly reliable, and the average error is $4.68 \%$ for subseries $\mathbf{X}_{1}$. The maximum error for the fusion true value is only $14.02 \%$, which appears in the $6^{\text {th }}$ step, and the maximum error before fusing is $14.68 \%$. The average error is $4.01 \%$ for subseries $\mathbf{X}_{2}$, and its maximum error for the fusing true value is only $6.82 \%$, appearing in the first step, and the maximum error before fusing is $11.43 \%$. The average error is $4.36 \%$ for subseries $\mathbf{X}_{3}$, and its maximum error for the fusing true value is only $7.85 \%$, appearing in the $5^{\text {th }}$ step, and the maximum error before fusing is as high as $12.70 \%$. The average error is $4.61 \%$ for subseries $\mathbf{X}_{4}$, and its maximum error for the fusing true value is only $10.58 \%$, appearing in the $9^{\text {th }}$ step, and the maximum error before fusing is as high as $13.63 \%$. Obviously, through using grey bootstrap and maximum entropy methods, the forecast errors for the fusing true value for each subseries decrease significantly, which indicates that the true value $X_{0}$ after fusion can better reflect the trend change in future service accuracy for superprecision rolling bearings, and the forecasting results are more accurate and feasible and can be better applied to practical engineering.

\subsection{Dynamic Prediction for Accuracy Maintaining Reliability.} The accuracy true value and interval have been accurately predicted for a superprecision rolling bearing for each step in the future, and on this basis, if we want to realize a prediction for accuracy maintaining reliability in each step, it is necessary to use the Poisson counting process: first, according to Section 3.2, there are 20,000 grey bootstrapgenerated data for the forecast values in each step; then setting the accuracy threshold $h=1.0 \mathrm{~m} \cdot \mathrm{s}^{-2}$ to the Poisson count (the value of the threshold $h$ depends on the bearing requirements levels of vibration performance of the main axis system, and the following will analyze it in detail), the number of $\mu$ outside the interval $[0,1.0]$ of optimum service accuracy is obtained for 20,000 generated data in each step, and the variation intensity $\theta$ is then acquired according to Equation (34), with the results shown in Table 4. Finally, dynamic prediction for accuracy maintaining reliability at each step can be achieved by using Equation (36), and the results are shown in Table 5.
From Table 4, it is not difficult to see that the number of $\mu$ exceeding the threshold $h$ is very similar or even equal in a single subseries. For the $\mathbf{X}_{1}$ and $\mathbf{X}_{4}$ subseries, the generated data for the former are all in the interval of optimum service accuracy, but those for the latter are all beyond the interval. For the $\mathbf{X}_{2}$ and $\mathbf{X}_{3}$ subseries, the number for the former in the interval of optimum service accuracy is around 25503925 and that for the latter is around 10065-12091. The difference in variation intensity $\theta$ is also small in the single subseries, and the variation intensity is 0 for subseries $\mathbf{X}_{1}$ in each step, which indicates that the bearing running conditions are very stable without any malignant variation at that time, and the running state is very good for maintaining optimum service accuracy. The variation intensity is 1 for subseries $\mathbf{X}_{4}$ in each step, which indicates that the bearing running condition is extremely poor, and the bearing may have incurred an accuracy failure. The variation intensity is between 0.1275 and 0.1963 for subseries $\mathbf{X}_{2}$ in each step, showing that the bearing is beginning to mutate, bearing running condition is becoming poor, and the probability of maintaining the optimum service status is gradually decreasing. In addition, this time point can provide a reference point for online health monitoring. The variation intensity is between 0.5033 and 0.6046 for subseries $\mathbf{X}_{3}$ in each step, showing that the bearing variation is serious, and the operating condition is very poor, so maintenance measures should be performed in time to avoid serious accidents. Therefore, the prediction results demonstrate good consistency for the single subseries. Meanwhile, subseries $\mathbf{X}_{1}, \mathbf{X}_{2}$, $\mathbf{X}_{3}$, and $\mathbf{X}_{4}$ stand for different running stages of a superprecision rolling bearing in service, showing that with the increase in operation time, the variation intensity will increase gradually, and the degree of malignant variation will be more serious, which results in a decline in the accuracy maintaining reliability.

From Table 5, the accuracy maintaining reliability can reach $100 \%$ for subseries $\mathbf{X}_{1}$ in the next 10 steps, and this is because the time series $\mathbf{X}_{1}$ is the initial stage of the experiment, the vibration amplitude and spindle runout are small with a high service accuracy, and the operation is very safe and reliable. The accuracy maintaining reliability is between 80 and $90 \%$ for subseries $\mathbf{X}_{2}$ in the next 10 steps, showing that accuracy maintaining reliability is general for the superprecision rolling bearing, and the possibility of maintaining an optimum service state is gradually decreasing. Thus, the bearing is gradually beginning to mutate, and a potential deterioration in accuracy is occurring in the internal part of the bearing. The accuracy maintaining reliability is between 50 and $80 \%$ for subseries $\mathbf{X}_{3}$ in the next 10 steps, showing that the accuracy reliability is poor for the superprecision rolling bearing, and the malignant variation is serious, so remedial measures should be taken in time. The reliability is less than $50 \%$ for subseries $\mathbf{X}_{4}$ in the next 10 steps, indicating that the superprecision rolling bearing is remarkably unreliable, and the bearing may have incurred an accuracy failure or even significant wear.

By analyzing the variation intensity and accuracy maintaining reliability at different time periods for $\mathbf{X}_{1}, \mathbf{X}_{2}$, $\mathbf{X}_{3}$, and $\mathbf{X}_{4}$, the dynamic evolution can be recognized 
TABLE 3: Estimated interval $\left[X_{\mathrm{L}}, X_{\mathrm{U}}\right]$ for each subseries for 10 steps in the future.

\begin{tabular}{lcccc}
\hline Step number & $\mathbf{X}_{1}$ & $\mathbf{X}_{2}$ & $\mathbf{X}_{3}$ & $\mathbf{X}_{4}$ \\
\hline $1^{\text {st }}$ step & {$[0.6677,0.7121]$} & {$[0.8308,1.0281]$} & {$[0.8296,1.2551]$} & {$[1.2267,1.4982]$} \\
$2^{\text {nd }}$ step & {$[0.6528,0.7254]$} & {$[0.8302,1.0249]$} & {$[0.8770,1.1254]$} & {$[1.1340,1.4847]$} \\
$3^{\text {rd }}$ step & {$[0.6526,0.7398]$} & {$[0.8156,1.0439]$} & {$[0.9121,1.1348]$} & {$[1.2325,1.4740]$} \\
$4^{\text {th }}$ step & {$[0.6673,0.7222]$} & {$[0.7984,1.0429]$} & {$[0.8487,1.1463]$} & {$[1.2192,1.4813]$} \\
$5^{\text {th }}$ step & {$[0.6656,0.6893]$} & {$[0.7836,1.0441]$} & {$[0.8788,1.1448]$} & {$[1.2566,1.4347]$} \\
$6^{\text {th }}$ step & {$[0.6738,0.6944]$} & {$[0.7632,1.0744]$} & {$[0.9342,1.1045]$} & {$[1.1931,1.4819]$} \\
$7^{\text {th }}$ step & {$[0.6326,0.7795]$} & {$[0.7579,1.0499]$} & {$[0.8698,1.1459]$} & {$[1.2153,1.5019]$} \\
$8^{\text {th }}$ step & {$[0.6685,0.6949]$} & {$[0.7979,1.0433]$} & {$[0.9256,1.1340]$} & {$[1.1741,1.5105]$} \\
$9^{\text {th }}$ step & {$[0.6383,0.7825]$} & {$[0.7867,1.0495]$} & {$[0.9059,1.1459]$} & {$[1.2446,1.4123]$} \\
$10^{\text {th }}$ step & {$[0.6312,0.7700]$} & {$[0.8202,1.0357]$} & {$[0.9029,1.1856]$} & {$[1.1871,1.4417]$} \\
\hline
\end{tabular}

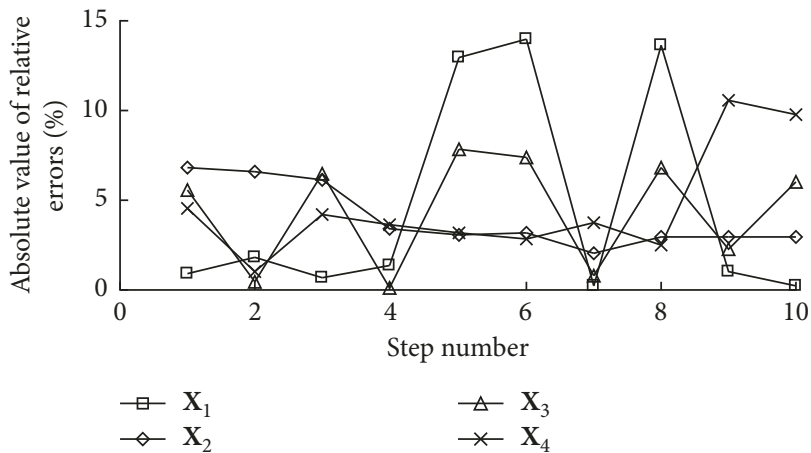

Figure 12: Prediction error of the estimated true value $X_{0}$ for each subseries.

TABle 4: Number of $\mu$ exceeding the threshold $h$ and variation intensity $\theta$.

\begin{tabular}{lcccccccc}
\hline \multirow{2}{*}{ Step number } & \multicolumn{2}{c}{$\mathbf{X}_{1}$} & \multicolumn{2}{c}{$\mathbf{X}_{2}$} & \multicolumn{2}{c}{$\mathbf{X}_{3}$} & \multicolumn{2}{c}{$\mathbf{X}_{4}$} \\
& $\mu$ & $\theta$ & $\mu$ & $\theta$ & $\mu$ & $\theta$ & $\mu$ & $\theta$ \\
\hline $1^{\text {st }}$ step & 0 & 0 & 3066 & 0.1533 & 11213 & 0.5607 & 20000 & 1 \\
$2^{\text {nd }}$ step & 0 & 0 & 2661 & 0.1331 & 11060 & 0.5530 & 20000 & 1 \\
$3^{\text {rd }}$ step & 0 & 0 & 3821 & 0.1911 & 11474 & 0.5737 & 20000 & 1 \\
$4^{\text {th }}$ step & 0 & 0 & 3007 & 0.1504 & 10065 & 0.5033 & 20000 & 1 \\
$5^{\text {th }}$ step & 0 & 0 & 2989 & 0.1495 & 10557 & 0.5279 & 20000 & 1 \\
$6^{\text {th }}$ step & 0 & 0 & 3730 & 0.1865 & 11705 & 0.5853 & 20000 & 1 \\
$7^{\text {th }}$ step & 0 & 0 & 3488 & 0.1744 & 10879 & 0.5440 & 20000 & 1 \\
$8^{\text {th }}$ step & 0 & 0 & 3153 & 0.1577 & 11530 & 0.5765 & 20000 & 1 \\
$9^{\text {th }}$ step & 0 & 0 & 3925 & 0.1963 & 11104 & 0.5552 & 20000 & 1 \\
$10^{\text {th }}$ step & 0 & 0 & 2550 & 0.1275 & 12091 & 0.6046 & 20000 & 1 \\
\hline
\end{tabular}

as illustrating the service accuracy of a superprecision rolling bearing. Compared with the total service length of $80010 \times 10 \mathrm{~min}$, the time of prediction for the future 10 steps can be viewed as instantaneous. The instantaneous reliability is the mean value of the prediction values over the next 10 steps, which stands for the accuracy maintaining reliability at the end of each subseries. The results are shown in Figure 13.

From Figure 13, the reliability at the end of each subseries can describe the trend variation in service accuracy well for a superprecision rolling bearing: at the end of each subseries $\mathbf{X}_{1}$, the accuracy maintaining reliability is large, showing that the possibility of maintaining an optimum service state is great, and the super-precision rolling bearing
TABLE 5: Accuracy maintaining reliability for the subseries at each step.

\begin{tabular}{lcccc}
\hline Step number & $\mathbf{X}_{1}(\%)$ & $\mathbf{X}_{2}(\%)$ & $\mathbf{X}_{3}(\%)$ & $\mathbf{X}_{4}(\%)$ \\
\hline $1^{\text {st }}$ step & 100 & 85.79 & 57.08 & 36.79 \\
$2^{\text {nd }}$ step & 100 & 87.54 & 57.52 & 36.79 \\
$3^{\text {rd }}$ step & 100 & 82.60 & 56.34 & 36.79 \\
$4^{\text {th }}$ step & 100 & 86.04 & 60.45 & 36.79 \\
$5^{\text {th }}$ step & 100 & 86.11 & 58.98 & 36.79 \\
$6^{\text {th }}$ step & 100 & 82.99 & 55.69 & 36.79 \\
$7^{\text {th }}$ step & 100 & 84.00 & 58.04 & 36.79 \\
$8^{\text {th }}$ step & 100 & 85.41 & 56.19 & 36.79 \\
$9^{\text {th }}$ step & 100 & 82.18 & 57.40 & 36.79 \\
$10^{\text {th }}$ step & 100 & 88.03 & 54.63 & 36.79 \\
\hline
\end{tabular}

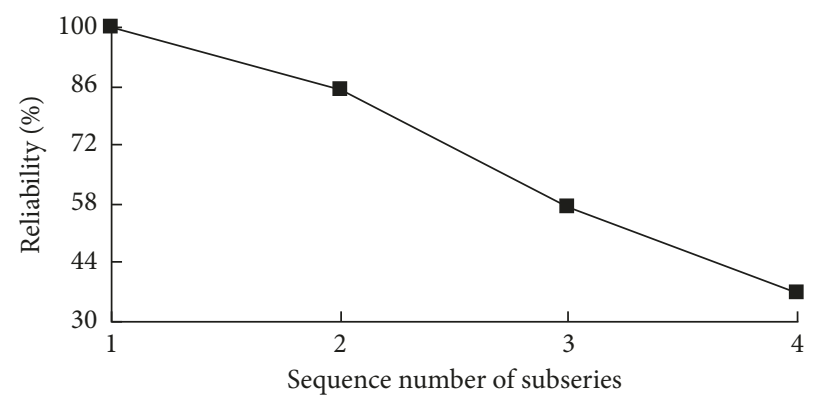

FIGURE 13: Accuracy maintaining reliability at the end of each subseries.

has a high running accuracy; at the end of each subseries $\mathbf{X}_{2}$, the reliability begins to decline, showing that the possibility of maintaining an optimum service state decreases gradually, and the running accuracy of the bearing is slowing down; at the end of each subseries $\mathbf{X}_{3}$, the reliability decreases rapidly, showing that the running accuracy of the bearing drops fast; and at the end of each subseries $\mathbf{X}_{4}$, the reliability reaches the minimum, showing that the service accuracy of the bearing has been lost already, and even fatigue failure has set in.

From Tables 4 and 5 and Figure 13, the accuracy threshold $h$ determines the variation intensity in service accuracy for each step in the future, thereby affecting the reliability of each step. However, the threshold value $h$ depends on the demanding degree of vibration performance for superprecision rolling bearings in machine tools, motors, or other systems. The accuracy maintaining reliability for 
TABLE 6: Accuracy maintaining reliability at different accuracy thresholds for subseries $\mathbf{X}_{1}$.

\begin{tabular}{lccccc}
\hline $\begin{array}{l}\text { Step } \\
\text { number }\end{array}$ & $\begin{array}{c}h=0.6 \\
(\%)\end{array}$ & $\begin{array}{c}h=0.8 \\
(\%)\end{array}$ & $\begin{array}{c}h=1.0 \\
(\%)\end{array}$ & $\begin{array}{c}h=1.2 \\
(\%)\end{array}$ & $\begin{array}{c}h=1.4 \\
(\%)\end{array}$ \\
\hline $1^{\text {st }}$ step & 36.79 & 100 & 100 & 100 & 100 \\
$2^{\text {nd }}$ step & 36.79 & 100 & 100 & 100 & 100 \\
$3^{\text {rd }}$ step & 36.79 & 100 & 100 & 100 & 100 \\
$4^{\text {th }}$ step & 36.79 & 100 & 100 & 100 & 100 \\
$5^{\text {th }}$ step & 36.79 & 100 & 100 & 100 & 100 \\
$6^{\text {th }}$ step & 36.79 & 100 & 100 & 100 & 100 \\
$7^{\text {th }}$ step & 36.79 & 100 & 100 & 100 & 100 \\
$8^{\text {th }}$ step & 36.79 & 100 & 100 & 100 & 100 \\
$9^{\text {th }}$ step & 36.79 & 100 & 100 & 100 & 100 \\
$10^{\text {th }}$ step & 36.79 & 100 & 100 & 100 & 100 \\
\hline
\end{tabular}

TABle 7: Accuracy maintaining reliability at different accuracy thresholds for subseries $\mathbf{X}_{2}$.

\begin{tabular}{lccccc}
\hline $\begin{array}{l}\text { Step } \\
\text { number }\end{array}$ & $\begin{array}{c}h=0.6 \\
(\%)\end{array}$ & $\begin{array}{c}h=0.8 \\
(\%)\end{array}$ & $\begin{array}{c}h=1.0 \\
(\%)\end{array}$ & $\begin{array}{c}h=1.2 \\
(\%)\end{array}$ & $\begin{array}{c}h=1.4 \\
(\%)\end{array}$ \\
\hline $1^{\text {st }}$ step & 36.79 & 36.79 & 85.79 & 100 & 100 \\
$2^{\text {nd }}$ step & 36.79 & 36.79 & 87.54 & 100 & 100 \\
$3^{\text {rd }}$ step & 36.79 & 36.79 & 82.60 & 100 & 100 \\
$4^{\text {th }}$ step & 36.79 & 36.79 & 86.04 & 100 & 100 \\
$5^{\text {th }}$ step & 36.79 & 39.59 & 86.11 & 100 & 100 \\
$6^{\text {th }}$ step & 36.79 & 39.62 & 82.99 & 100 & 100 \\
$7^{\text {th }}$ step & 36.79 & 40.69 & 84.00 & 100 & 100 \\
$8^{\text {th }}$ step & 36.79 & 36.79 & 85.41 & 100 & 100 \\
$9^{\text {th }}$ step & 36.79 & 37.86 & 82.18 & 100 & 100 \\
$10^{\text {th }}$ step & 36.79 & 36.79 & 88.03 & 100 & 100 \\
\hline
\end{tabular}

TABLE 8: Accuracy maintaining reliability at different accuracy thresholds for subseries $\mathbf{X}_{3}$.

\begin{tabular}{lccccc}
\hline $\begin{array}{l}\text { Step } \\
\text { number }\end{array}$ & $\begin{array}{c}h=0.6 \\
(\%)\end{array}$ & $\begin{array}{c}h=0.8 \\
(\%)\end{array}$ & $\begin{array}{c}h=1.0 \\
(\%)\end{array}$ & $\begin{array}{c}h=1.2 \\
(\%)\end{array}$ & $\begin{array}{c}h=1.4 \\
(\%)\end{array}$ \\
\hline $1^{\text {st }}$ step & 36.79 & 36.79 & 57.08 & 90.56 & 100 \\
$2^{\text {nd }}$ step & 36.79 & 36.79 & 57.52 & 100 & 100 \\
$3^{\text {rd }}$ step & 36.79 & 36.79 & 56.34 & 100 & 100 \\
$4^{\text {th }}$ step & 36.79 & 36.79 & 60.45 & 100 & 100 \\
$5^{\text {th }}$ step & 36.79 & 36.79 & 58.98 & 100 & 100 \\
$6^{\text {th }}$ step & 36.79 & 36.79 & 55.69 & 100 & 100 \\
$7^{\text {th }}$ step & 36.79 & 36.79 & 58.04 & 100 & 100 \\
$8^{\text {th }}$ step & 36.79 & 36.79 & 56.19 & 100 & 100 \\
$9^{\text {th }}$ step & 36.79 & 36.79 & 57.40 & 100 & 100 \\
$10^{\text {th }}$ step & 36.79 & 36.79 & 54.63 & 100 & 100 \\
\hline
\end{tabular}

TABLE 9: Accuracy maintaining reliability at different accuracy thresholds for subseries $\mathbf{X}_{4}$.

\begin{tabular}{lccccc}
\hline $\begin{array}{l}\text { Step } \\
\text { number }\end{array}$ & $\begin{array}{c}h=0.6 \\
(\%)\end{array}$ & $\begin{array}{c}h=0.8 \\
(\%)\end{array}$ & $\begin{array}{c}h=1.0 \\
(\%)\end{array}$ & $\begin{array}{c}h=1.2 \\
(\%)\end{array}$ & $\begin{array}{c}h=1.4 \\
(\%)\end{array}$ \\
\hline $1^{\text {st }}$ step & 36.79 & 36.79 & 36.79 & 36.79 & 65.20 \\
$2^{\text {nd }}$ step & 36.79 & 36.79 & 36.79 & 53.14 & 67.62 \\
$3^{\text {rd }}$ step & 36.79 & 36.79 & 36.79 & 36.79 & 65.38 \\
$4^{\text {th }}$ step & 36.79 & 36.79 & 36.79 & 36.79 & 68.21 \\
$5^{\text {th }}$ step & 36.79 & 36.79 & 36.79 & 36.79 & 80.41 \\
$6^{\text {th }}$ step & 36.79 & 36.79 & 36.79 & 36.79 & 70.08 \\
$7^{\text {th }}$ step & 36.79 & 36.79 & 36.79 & 36.79 & 62.19 \\
$8^{\text {th }}$ step & 36.79 & 36.79 & 36.79 & 39.46 & 65.26 \\
$9^{\text {th }}$ step & 36.79 & 36.79 & 36.79 & 36.79 & 92.70 \\
$10^{\text {th }}$ step & 36.79 & 36.79 & 36.79 & 37.65 & 82.21 \\
\hline
\end{tabular}

subseries $\mathbf{X}_{1}, \mathbf{X}_{2}, \mathbf{X}_{3}$, and $\mathbf{X}_{4}$ is discussed by using different accuracy thresholds in Tables 6-9.

According to the prediction series for accuracy maintaining reliability under different accuracy thresholds $h$ from Tables 6-9, it can be seen that the smaller the accuracy threshold is, the lower the reliability for the next step is during the dynamic prediction process for accuracy maintaining reliability for each subseries; conversely, the higher the reliability is. The value size of the threshold reflects the bearing accuracy sensitivity of the spindle system. Therefore, in practice, the corresponding accuracy threshold $h$ should be designed in advance according to the spindle system requirements levels for vibration performance of a superprecision rolling bearing. Real-time monitoring of accuracy information and then obtaining the corresponding reliability can discover failures and hidden dangers in time and thus avoid bad accidents.

3.4. Service Accuracy Maintaining the Relative Reliability. From Tables 6-9, it is not difficult to see that the prediction reliability for time series $\mathbf{X}_{1}$ is the highest under the same threshold value for the four subseries. Thus, the best period for maintaining an optimum service accuracy is the time interval $\mathbf{X}_{1}$ for superprecision rolling bearings, where accuracy maintaining reliability is $R\left(\theta_{1}\right)$, and the accuracy maintaining reliability of the other subseries $\mathbf{X}_{2}, \mathbf{X}_{3}$, and $\mathbf{X}_{4}$ is $R\left(\theta_{2}\right), R\left(\theta_{3}\right)$, and $R\left(\theta_{4}\right)$ according to Section 2.4.3, respectively. Taking $h=1.0 \mathrm{~m} \cdot \mathrm{s}^{-2}$ and $h=1.2 \mathrm{~m} \cdot \mathrm{s}^{-2}$ as an example to analyze the relative reliability of maintaining service accuracy, when $h=1.0 \mathrm{~m} \cdot \mathrm{s}^{-2}$, the mean values for prediction reliability are solved for each subseries in the next 10 steps, obtaining $R\left(\theta_{1}\right)=100 \%, R\left(\theta_{2}\right)=85.07 \%, R\left(\theta_{3}\right)=$ $57.23 \%$, and $R\left(\theta_{4}\right)=36.79 \%$; when $h=1.2 \mathrm{~m} \cdot \mathrm{s}^{-2}$, obtaining $R\left(\theta_{1}\right)=100 \%, R\left(\theta_{2}\right)=100 \%, R\left(\theta_{3}\right)=99.06 \%$, and $R\left(\theta_{4}\right)=$ $38.78 \%$. With Equation (37), the degree of failure $d(\eta)$ for accuracy maintaining an optimum service state is acquired for superprecision rolling bearings at different service time intervals, and the results are shown in Figure 14.

From Figure 14, when the accuracy threshold $h=$ $1.0 \mathrm{~m} \cdot \mathrm{s}^{-2}$ and $\eta=2$, the relative reliability is $d(\eta)=0 \%$ at the end of subseries $\mathbf{X}_{2}$, which shows that the accuracy reliability at the end of subseries $\mathbf{X}_{2}$ is not less than its reliability at its best period of service, and the bearing service accuracy is undeniably at its optimal state at this time. When $\eta=3$, the relative reliability is $d(\eta)=-0.94 \% \in[-10 \%, 0 \%)$ at the end of subseries $\mathbf{X}_{3}$ and $d(\eta)$ is close to $0 \%$, which shows that the service accuracy at this time is normal, and the failure probability of maintaining an optimal accuracy state is low; viz., its service accuracy is close to the optimal state. When $\eta=4$, the relative reliability is $d(\eta)=-61.22 \%<-20 \%$ at the end of subseries $\mathbf{X}_{4}$, which shows that the service accuracy at this time is very poor, the failure probability of maintaining an optimal accuracy state is quite high, and the service accuracy of the bearing may have failed. When the accuracy threshold $h=1.2 \mathrm{~m} \cdot \mathrm{s}^{-2}$ and $\eta=2$, the relative reliability is $d$ $(\eta)=-14.93 \% \in[-20 \%,-10 \%)$ at the end of subseries $\mathbf{X}_{2}$, showing that the service accuracy at this time becomes gradually worse, and the failure probability of maintaining 


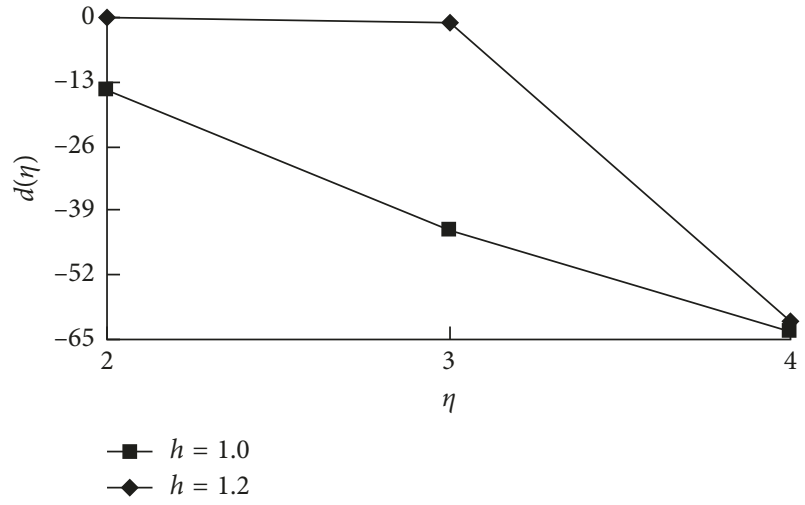

FIgURE 14: Accuracy maintaining the relative reliability for superprecision rolling bearings.

an optimal accuracy state increases gradually; when $\eta=3$ and $\eta=4$, the relative reliability is $d(\eta)=$ $-42.77 \%<-20 \%$ and $d(\eta)=-63.21 \%<-20 \%$ at the end of subseries $\mathbf{X}_{3}$ and $\mathbf{X}_{4}$, respectively, showing that the service accuracy is very poor, the failure probability of maintaining an optimal accuracy state is quite high, and the service accuracy of the bearing may have failed. The point in time for relative reliability $d(\eta)=-20 \%$ is the critical point at which the service accuracy begins to worsen. Thus, as a matter of practical engineering, checking and repairing the bearing in time and then taking appropriate remedial measures are necessary steps before reaching a critical moment for a bearing in service. This can avoid serious safety accidents caused by a failure in accuracy for a superprecision rolling bearing.

In summary, during the process of chaotic forecasting for time series, the adding-weight zero-rank local-region method, one-rank local-region method, adding-weight one-rank localregion method, and improved adding-weight one-rank localregion method are all feasible and accurate, with the maximum prediction error being no more than $15 \%$, thus meeting general forecasting requirements in engineering practice. Combining the grey bootstrap method with the maximum entropy principle to fuse the four prediction values of each step effectively, the future accuracy true value and estimated interval are obtained for a superprecision rolling bearing. According to the Poisson counting process and grey bootstrap-generated data, a dynamic prediction for accuracy maintaining reliability is achieved for superprecision rolling bearings for each step in the future, and the prediction results can effectively monitor the variation information on bearing service accuracy. Finally, a new concept is proposed for relative reliability of maintaining accuracy, which can effectively predict the degree of failure for maintaining an optimal accuracy state for a bearing.

\section{Conclusions}

(1) By fusing chaotic prediction models into the grey bootstrap principle and maximum entropy principle, the accuracy true value and fluctuation interval can be precisely predicted for superprecision rolling bearings for each step into the future. The prediction values and actual values demonstrate good consistency, and the prediction error is very small with a maximum relative error of only $14.02 \%$.

(2) Combining the grey bootstrap principle with the Poisson process, a dynamic prediction method is proposed for accuracy maintaining reliability in superprecision rolling bearings, which can realize the prediction of accuracy maintaining reliability for each step into the future and can reveal the mechanism of influence between the running time and service accuracy.

(3) According to the varying curves of accuracy maintaining reliability at different time intervals and the influence of threshold accuracy on reliability analysis, the evolution information for accuracy maintaining reliability can be predicted in real time for superprecision rolling bearings, and failures or hidden troubles can therefore be discovered in time.

(4) Based on the novel concept of accuracy maintaining reliability for superprecision rolling bearings, the possibility and degree of failure in maintaining an optimal accuracy state are predicted effectively. Some intervention measures for maintenance or replacement can be implemented in a timely manner before the possibility of failure in service accuracy becomes critical.

(5) The proposed models can not only realize dynamic prediction of accuracy maintaining reliability for superprecision rolling bearings at each point in time but also realize an estimation of true value and interval for the accuracy attribute.

\section{Data Availability}

The data used to support the findings of this study are included within the article.

\section{Conflicts of Interest}

The authors declare that there are no conflicts of interest regarding the publication of this paper.

\section{Acknowledgments}

This project was supported by the National Natural Science Foundation of China (Grant No. 51475144) and Natural Science Foundation of Henan Province of China (Grant No. 162300410065).

\section{References}

[1] A. Khosravi and Y. S. Kavian, "Autonomous fault-diagnosis and decision-making algorithm for determining faulty nodes in distributed wireless networks," Frontiers of Information Technology \& Electronic Engineering, vol. 17, no. 9, pp. 885896, 2016.

[2] S. Negi and S. B. Singh, "Reliability analysis of non-repairable complex system with weighted subsystems connected in 
series," Applied Mathematics \& Computation, vol. 262, pp. 79-89, 2015.

[3] X. T. Xia, "Reliability analysis of zero-failure data with poor information," Quality and Reliability Engineering International, vol. 28, no. 8, pp. 981-990, 2012.

[4] B. Zhang, L. J. Zhang, and J. W. Xu, "Degradation feature selection for remaining useful life prediction of rolling element bearings," Quality \& Reliability Engineering International, vol. 32, no. 2, pp. 547-554, 2016.

[5] S. Menon, X. H. Jin, T. W. S. Chow, and M. Pecht, "Evaluating covariance in prognostic and system health management applications," Mechanical Systems and Signal Processing, vol. 58-59, pp. 206-217, 2015.

[6] P. Jiang, Y. Y. Xing, X. Jia, and B. Guo, "Weibull failure probability estimation based on zero-failure data," Mathematical Problems in Engineering, vol. 2015, Article ID 681232, 8 pages, 2015.

[7] R. B. Bergmann and A. Bill, "On the origin of logarithmicnormal distributions: an analytical derivation, and its application to nucleation and growth processes," Journal of Crystal Growth, vol. 310, no. 13, pp. 3135-3138, 2008.

[8] A. Mahmoud, "Economic allocation of reliability growth testing using Weibull distributions," Reliability Engineering and System Safety, vol. 152, pp. 273-280, 2016.

[9] H. Kim, S. H. Lee, J. S. Park, H. Kim, Y. S. Chang, and G. Heo, "Reliability data update using condition monitoring and prognostics in probabilistic safety assessment," Nuclear Engineering and Technology, vol. 47, no. 2, pp. 204-211, 2015.

[10] D. An, N. H. Kim, and J. H. Choi, "Practical options for selecting data-driven or physics-based prognostics algorithms with reviews," Reliability Engineering and System Safety, vol. 133, pp. 223-236, 2015.

[11] K. Liu, N. Z. Gebraeel, and J. Shi, “A data-level fusion model for developing composite health indices for degradation modeling and prognostic analysis," IEEE Transactions on Automation Science and Engineering, vol. 10, no. 3, pp. 652664, 2013.

[12] L. Bian and N. Gebraeel, "Computing and updating the firstpassage time distribution for randomly evolving degradation signals," IIE Transactions, vol. 44, no. 11, pp. 974-987, 2012.

[13] J. M. Karandikar, N. H. Kim, and T. L. Schmitz, "Prediction of remaining useful life for fatigue-damaged structures using Bayesian inference," Engineering Fracture Mechanics, vol. 96, no. 2, pp. 588-605, 2012.

[14] S. Hong, Z. Zhou, E. Zio, and W. B. Wang, "An adaptive method for health trend prediction of rotating bearings," Digital Signal Processing, vol. 35, pp. 117-123, 2014.

[15] X. D. Tan, J. L. Luo, Q. Li, B. Lu, and J. Qiu, "Fault evolutiontest dependency modeling for mechanical systems," Frontiers of Information Technology \& Electronic Engineering, vol. 16, no. 10, pp. 848-857, 2015.

[16] X. T. Xia, "Forecasting method for product reliability along with performance data," Journal of Failure Analysis and Prevention, vol. 12, no. 5, pp. 532-540, 2012.

[17] F. M. Huang, J. S. Huang, S. H. Jiang, and C. B. Zhou, "Landslide displacement prediction based on multivariate chaotic model and extreme learning machine," Engineering Geology, vol. 218, pp. 173-186, 2017.

[18] G. Heydari, M. A. Vali, and A. A. Gharaveisi, "Chaotic time series prediction via artificial neural square fuzzy inference system," Expert Systems with Applications, vol. 55, pp. 461468, 2016.

[19] L. M. Yang, W. Zhang, and Y. F. Chen, "Time-series prediction based on global fuzzy measure in social networks,"
Frontiers of Information Technology \& Electronic Engineering, vol. 16, no. 10, pp. 805-816, 2015.

[20] Y. S. Chen, J. L. Yang, Y. H. Xu, S. D. Jiang, X. D. Liu, and Q Wang, "Status self-validation of sensor arrays using gray forecasting model and bootstrap method," IEEE Transactions on Instrumentation \& Measurement, vol. 65, no. 7, pp. 1626-1640, 2016.

[21] X. T. Xia, X. Y. Chen, and Y. F. Chen, "Grey bootstrap method of evaluation of uncertainty in dynamic measurement," Measurement, vol. 41, no. 6, pp. 687-696, 2008.

[22] A. Tagliani, "Hausdorff moment problem and maximum entropy: on the existence conditions," Applied Mathematics and Computation, vol. 231, pp. 111-116, 2014.

[23] A. Giffin, C. Cafaro, and S. A. Ali, "Application of the maximum relative entropy method to the physics of ferromagnetic materials," Physica A: Statistical Mechanics and its Applications, vol. 455, pp. 11-26, 2016.

[24] A. Moscovich and B. Nadler, "Fast calculation of boundary crossing probabilities for Poisson processes," Statistics and Probability Letters, vol. 123, pp. 177-182, 2016.

[25] A. S. Andreou and S. P. Chatzis, "Software defect prediction using doubly stochastic Poisson processes driven by stochastic belief networks," Journal of Systems and Software, vol. 122, pp. 72-82, 2016.

[26] J. L. Deng, "Introduction to grey system theory," Journal of Grey System, vol. 1, no. 1, pp. 1-24, 1989. 


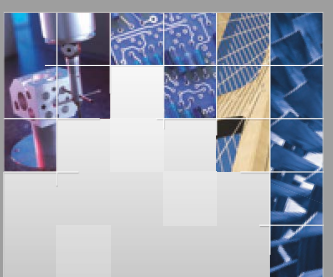

\section{Enfincering}
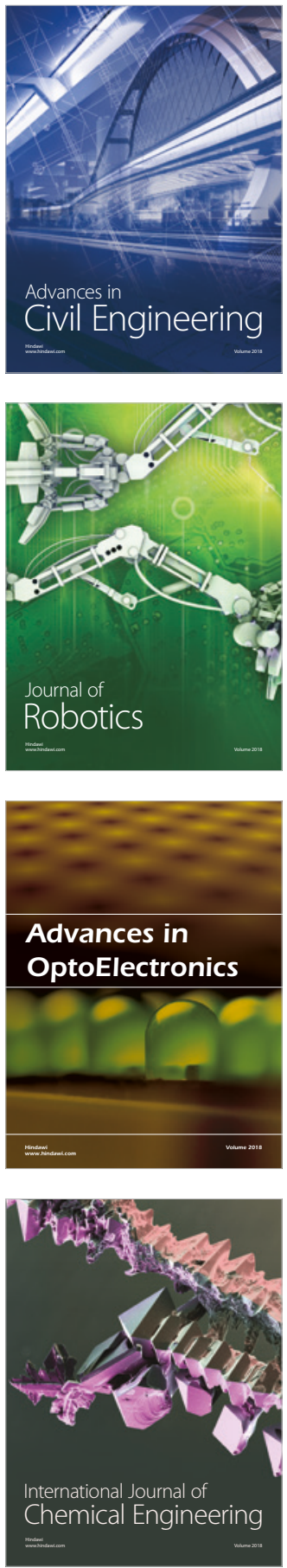

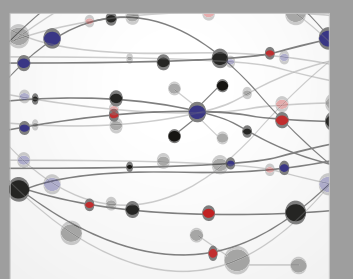

\section{Rotating \\ Machinery}

The Scientific World Journal

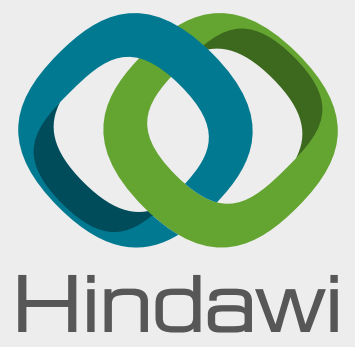

Submit your manuscripts at

www.hindawi.com
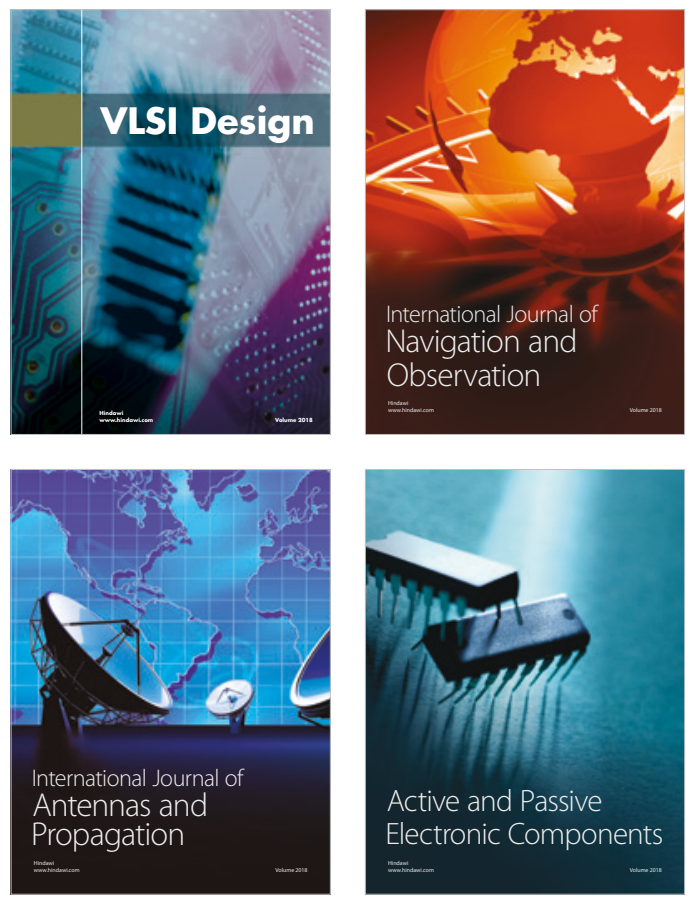
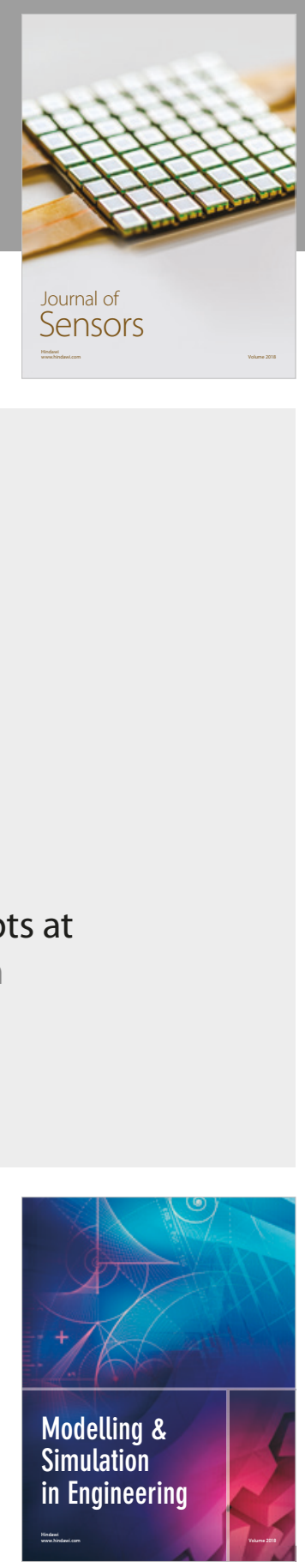

\section{Advances \\ Multimedia}
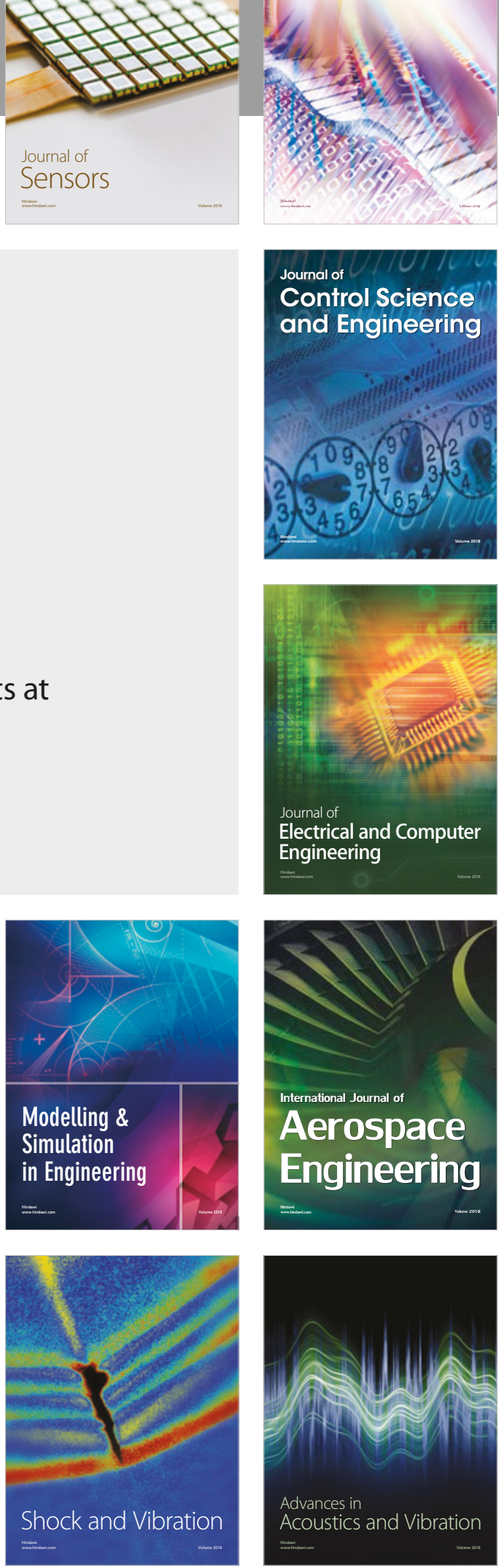